Luca Capogna $\cdot$ Nicola Garofalo

\title{
Regularity of minimizers of the calculus of variations in Carnot groups via hypoellipticity of systems of Hörmander type
}

\author{
Received October 23, 2000 / final version received February 5, 2002 \\ Published online May 15, 2002 - (C) Springer-Verlag \& EMS 2002
}

\begin{abstract}
We prove the hypoellipticity for systems of Hörmander type with constant coefficients in Carnot groups of step 2. This result is used to implement blow-up methods and prove partial regularity for local minimizers of non-convex functionals, and for solutions of non-linear systems which appear in the study of non-isotropic metric structures with scalings. We also establish estimates of the Hausdorff dimension of the singular set.
\end{abstract}

\section{Introduction}

The present paper constitutes the first part of a project aimed at developing the regularity theory for local minimizers of non-convex variational functionals and for weak solutions of quasi-linear systems in a class of non-isotropic spaces known as Carnot groups. Here, we focus on the non-evolutive case and on the model situation of groups of step 2. In a series of forthcoming articles we will study the technically more involved case of Carnot groups of arbitrary step, and we will also prove partial regularity results for quasi-linear parabolic systems which arise in the study of the harmonic maps flow. We will also consider the regularity theory for variational functionals and systems with special structure.

A Carnot group of step $r \geq 1$ is a simply connected Lie group $\mathbf{G}$ whose Lie algebra admits a vector space decomposition in $r$ layers $\mathbf{g}=V^{1} \oplus \ldots \oplus V^{r}$ having the two properties: (i) $\mathbf{g}$ is stratified, i.e., $\left[V^{1}, V^{j}\right]=V^{j+1}, j=1, \ldots, r-1$; (ii) $\mathbf{g}$ is $r$-nilpotent, that is, $\left[V^{j}, V^{r}\right]=\{0\}, j=1, \ldots, r$. We note that the exponential map exp : $\mathbf{g} \rightarrow \mathbf{G}$ defines a global diffeomorphism. Such a group is equipped with a natural family of non-isotropic dilations. One starts with defining dilations $\Delta_{\lambda}$ on the Lie algebra as follows. If $X=X_{1}+\ldots+X_{r} \in \mathbf{g}$, with $X_{j} \in V^{j}$, $j=1, \ldots, r$, then $\Delta_{\lambda}(X)=\lambda X_{1}+\ldots+\lambda^{r} X_{r}$. The group dilations $\delta_{\lambda}: \mathbf{G} \rightarrow \mathbf{G}$ are then introduced by lifting $\Delta_{\lambda}$ via the exponential map $\exp : \mathbf{g} \rightarrow \mathbf{G}$, i.e.,

L. Capogna: Department of Mathematics, University of Arkansas, Fayetteville, AR 72701, USA; e-mail: lcapogna@comp.uark.edu

The work of the first author was partially supported by NSF Grant No. DMS-9800794

N. Garofalo: Department of Mathematics, The Johns Hopkins University, Baltimore, MD 21218, USA; e-mail: ngarofalo@math.jhu.edu

The work of the second author was partially supported by NSF Grants No. DMS-9706892 and DMS-0070492 
$\delta_{\lambda}(x)=\exp \circ \Delta_{\lambda} \circ \exp ^{-1}(x)$. We denote by $d x$ the bi-invariant Haar measure on $\mathbf{G}$ obtained by pushing forward the standard Lebesgue measure on $\mathbf{g}$ via the exponential map. When we say that a set has measure zero in $\mathbf{G}$ we mean this with respect to $d x$. One easily recognizes that $\left(d \circ \delta_{\lambda}\right) x=\lambda^{Q} d x$, where the number

$$
Q=\sum_{j=1}^{r} j \operatorname{dim}\left(V^{j}\right)
$$

is the so-called homogeneous dimension of $\mathbf{G}$. Such number is larger than the topological dimension $\sum_{j=1}^{r} \operatorname{dim}\left(V^{j}\right)$ of the group.

The homogeneous structure of $\mathbf{G}$ generated by the group dilations $\left\{\delta_{\lambda}\right\}_{\lambda>0}$ plays an important role in the analysis developed in this paper. Another aspect which will be equally important is the pseudo-metric structure which one associates to the stratification of $\mathbf{g}$ in the following natural way. We assume that $\mathbf{g}$ is equipped with an inner product $\langle\cdot, \cdot\rangle$ with respect to which the $V^{j}$ 's are mutually orthogonal, and let $|\cdot|=(<\cdot, \cdot>)^{1 / 2}$. For $X=X_{1}+\ldots+X_{r} \in \mathbf{g}$ one defines

$$
\|X\|_{\mathbf{g}}=\left(\sum_{i=1}^{r}\left|X_{i}\right|^{2 r ! / i}\right)^{2 r !},
$$

and

$$
\|x\|_{\mathbf{G}}=\|X\|_{\mathbf{g}} \quad \text { if } \quad x=\exp X .
$$

Henceforth, we will ordinarily omit the subscripts and simply write $\|x\|$ and $\|X\|$. The homogeneous pseudo-norm on $\mathbf{G}$ induces the following pseudo-distance $d(x, y)=\left\|x^{-1} y\right\|$. One has for every $x, y, z \in \mathbf{G}$ and for any $\lambda>0$

$$
d(z x, z y)=d(x, y), \quad d\left(\delta_{\lambda}(x), \delta_{\lambda}(y)\right)=\lambda d(x, y) .
$$

Using these properties it is easy to recognize that the Haar measure of $B(x, R)=$ $\{y \in \mathbf{G} \mid d(x, y)<R\}$ is given by $|B(x, R)|=\omega_{\mathbf{G}} R$, where $\omega_{\mathbf{G}}=|B(e, 1)|$ and $e$ is the group identity. This growth of the pseudo-balls accounts for the role of a critical dimension played by the number $Q$ in Theorems 1.1 and 1.2.

Carnot groups indirectly owe their name to the foundational paper of Carathéodory [Ca] on Carnot thermodynamics. Such Lie groups are similar to Euclidean spaces (which are themselves abelian Carnot groups with trivial step 1) in that they possess translations and dilations, and come with a measure which is invariant with respect to these operations. They also have a pseudo-metric structure, but when the step $r>1$, then the non-abelian group law produces a strong distortion of the underlying geometry. As a consequence, the basic properties of the latter are extremely different, and much harder to grasp, then those of Euclidean spaces. There exists a very wide variety of situations in which Carnot groups appear as the natural setting, and it is impossible to attempt here even a brief description. Besides Carathéodory's cited paper, we mention the famous accessibility paper of Chow [Cho], which in many respects paved the way to the modern developments. In connection with optimal control theory and sub-Riemannian geometry one 
should also see the articles [Br] and [Su], and the recent book [BR]. In partial differential equations Carnot groups first implicitly appeared in Kolmogorov's short note $[\mathrm{Ko}]$. Thirty years later Hörmander $[\mathrm{H}]$ proved his fundamental theorem stating that, given a system $X_{o}, X_{1}, \ldots, X_{m}$ of smooth vector fields in $\mathbb{R}^{n}$, the condition $\operatorname{rank} \operatorname{Lie}\left\{X_{o}, X_{1}, \ldots, X_{m}\right\} \equiv n$ is sufficient for the hypoellipticity of the partial differential operator $L=\sum_{j=1}^{m} X_{j}^{2}+X_{o}$. These operators include trivially all elliptic and parabolic operators (with smooth coefficients), but in the important case when $m<n-1$, they define a class which fails to be either elliptic or parabolic. For instance, Kolmogorov's equation for the probability density of a system with $2 n$ degrees of freedom is precisely of this type. Here is the basic model in $\mathbb{R}^{3}: X_{1}^{2} u+X_{o} u=0$, where $X_{1}=\partial / \partial x$ and $X_{o}=-x \partial / \partial y-\partial / \partial t$, and one has $\left[X_{1}, X_{o}\right]=-\partial / \partial y$. Another operator, which is of paramount importance, is the real part of the Kohn sub-Laplacian on the Heisenberg group $\mathbb{H}^{n}$. In the three-dimensional model $\mathbb{H}^{1}$ such operator is given by $L=X_{1}^{2}+X_{2}^{2}$, where $X_{1}=\partial / \partial x+2 y \partial / \partial t, X_{2}=\partial / \partial y-2 x \partial / \partial t$, and one has the commutation relation $\left[X_{1}, X_{2}\right]=-4 \partial / \partial t$. The Heisenberg group is the most important Carnot group of step 2. For its description we refer the reader to section two. Carnot groups are the natural osculating spaces (or blow-up limits) for Cauchy-Riemann [FS], [S], and Carnot-Carathéodory manifolds [Pa], [Gr], [GN], [HK], [JX], [Che], [AK1], [AK2], [Ga]. In continuum mechanics they occur as limits of crystalline structures [Chr], but they also play an important role in other applied sciences, such as mechanical engineering or robotics $[\mathrm{CK}]$. These Lie groups are thus the natural testing ground for problems in Calculus of Variations which arise in the study of non-isotropic metric structures with scalings. In this paper we focus on the more elementary case of groups of step 2. The reason for this choice is that the study of these models incorporates most of the essential ideas. Many of the geometric obstructions of the more general situation are already present for groups of step 2, but the technical aspect is considerably less complicated.

Given a Carnot group of step 2 with Lie algebra $\mathbf{g}=V^{1} \oplus V^{2}$, we fix an orthonormal basis $X=\left\{X_{1}, \ldots, X_{m}\right\}$ of the first layer $V^{1}$ of $\mathbf{g}$. Thanks to the stratification assumption, the elements of $X$ and their brackets generate the whole algebra. We continue to use the same symbols $X_{j}$ for the corresponding leftinvariant vector fields on $\mathbf{G}$. For a function $u=\left(u^{1}, \ldots, u^{N}\right): \mathbf{G} \rightarrow \mathbb{R}^{N}$, the matrix $\{X u\}_{i, j}=X_{i} u^{j}$ denotes the Jacobian matrix of $u$ with respect to such basis. Since $\{X u\}$ represents differentiation only with respect to a basis of the first layer $V^{1}$, we will call it the horizontal Jacobian matrix of $u$. The main objective of this paper is to establish regularity properties in the non-horizontal (commutators) directions starting from structural assumptions which involve only control of the horizontal ones.

One of our main purposes is to study the partial regularity of local minimizers of functionals of the type

$$
\mathcal{F}(u, \Omega)=\int_{\Omega} F(X u) d p,
$$

where $F: \mathbb{R}^{m N} \rightarrow \mathbb{R}^{+}$is a $C^{2}$ function satisfying the following structural assumptions: 
For some $2 \leq q<\infty$, one has for every $\xi, \eta \in \mathbb{R}^{m N}$

$$
\begin{gathered}
F(\xi) \leq C\left(1+|\xi|^{q}\right), \\
\sum_{\alpha, \beta=1}^{N} \sum_{i, j=1}^{m} \partial_{\xi_{i}^{\alpha}} \partial_{\xi_{j}^{\beta}} F(\xi) \eta_{i}^{\alpha} \eta_{j}^{\beta} \geq \lambda(1+|\xi|)^{q-2}|\eta|^{2}>0 .
\end{gathered}
$$

Observe that while $F$ is strictly convex as a function of $X u$, it may fail to be so when viewed as a function of $\nabla u$ (the full Riemannian gradient of $u$ ). The prototype of the functionals we consider is the regularized horizontal $q$-energy

$$
E_{q}^{\varepsilon}(u, \Omega)=\int_{\Omega}\left(\varepsilon+|X u|^{2}\right)^{\frac{q}{2}} d p
$$

with $2 \leq q<\infty$. Here, $\Omega$ denotes a given open subset of $\mathbf{G}$.

Another objective of this paper is to prove the partial regularity of weak solutions of quasi-linear systems of the form

$$
-\sum_{\beta=1}^{N} \sum_{i, j=1}^{m} X_{i}\left(A_{i j}^{\alpha \beta}(x, u) X_{j} u^{\beta}\right)=f^{\alpha}(x, u, X u), \alpha=1, \ldots, N
$$

where $A_{i j}^{\alpha \beta}(x, u)$ are Hölder continuous functions satisfying together with $f=$ $\left(f^{1}, \ldots, f^{N}\right)$ the hypothesis: For every $M>0$ there exist $\lambda(M), a(M)>0$, such that for $x \in \Omega$ and $|u| \leq M$ one has

$$
\sum_{\alpha, \beta=1}^{N} \sum_{i, j=1}^{m} A_{i j}^{\alpha \beta}(x, u) \xi_{i}^{\alpha} \xi_{j}^{\beta} \geq \lambda(M)|\xi|^{2}
$$

and

$$
|f(x, u, \xi)| \leq a(M)|\xi|^{2}+b .
$$

The model for (1.3) is the sub-elliptic harmonic map system for mappings $u: \Omega \rightarrow N$, where $N$ is a Riemannian manifold. In this case the principal part of the operator involved is the sub-Laplacian on $\mathbf{G}$

$$
\mathcal{L}=\sum_{j=1}^{m} X_{j}^{2}
$$

The Dirichlet problem for such maps with suitable conditions on the target has been recently studied by Jost and Xu [JX] (see also [HS]). The parabolic version of (1.3) has clearly a geometric interest in view of the applications to the harmonic flow. This case will be studied in a sub-sequent article.

The regularity of local minimizers of (1.1) and of weak solutions to (1.3) will be measured in terms of the horizontal Hölder and Sobolev spaces introduced by Folland and Stein [FS], [F]. Denote by $H^{s}$ the $s$-dimensional Hausdorff measure generated by the pseudo-metric $d$. We prove the following. 
Theorem 1.1 Let $\mathbf{G}$ be a Carnot group of step two, and let $\Omega \subset \mathbf{G}$ be a bounded open set. If $u$ is a local minimizer of (1.1) in $\Omega$, then there exists a closed set $S \subset \Omega$, having measure zero, such that Xu is Hölder continuous in $\Omega \backslash S$. Moreover, if $q=2$ then $H^{Q-2+\sigma}(S)=0$, for every $\sigma>0$.

Theorem 1.2 Let $\mathbf{G}$ be a Carnot group of step two, and let $\Omega \subset \mathbf{G}$ be a bounded open set. If $u$ is a weak solution of (1.3) in $\Omega$, with the assumptions (1.4), (1.5), and if $a(M) M \leq \lambda(M) / 2$, then there exists a closed set $S \subset \Omega$ such that Xu is Hölder continuous in $\Omega \backslash S$, and $H^{Q-2+\sigma}(S)=0$, for every $\sigma>0$.

In the classical elliptic case corresponding results have been proved by various authors using either the blow-up technique or the approach of Morrey-Campanato (see [Mo], [GM], [GG1], [GG2], [Gi], [E]). The central result, on which both approaches rest, are the $W^{2,2}$-estimates for constant coefficient systems. When we tried to extend the classical theory to the non-elliptic setting of Carnot groups we were confronted with a major obstruction: The $W^{2,2}$-estimates for the corresponding constant coefficient systems of Hörmander type were lacking. The main contribution of the present paper consists in developing a direct proof of such estimates in the setting of Carnot groups of step two. After completing this part we will suitably modify and adapt several parts of either of the above mentioned approaches to the non-abelian setting.

The constant coefficient systems of interest for us are of the form

$$
-\sum_{\beta=1}^{N} \sum_{i, j=1}^{m} X_{i}\left(A_{i j}^{\alpha \beta} X_{j} u^{\beta}+f_{i}^{\alpha}\right)=f^{\alpha}, \alpha=1, \ldots, N
$$

where $A_{i j}^{\alpha \beta}$ is a tensor satisfying the coercivity condition on the horizontal directions

$$
\sum_{\alpha, \beta=1}^{N} \sum_{i, j=1}^{m} A_{i j}^{\alpha \beta} \xi_{i}^{\alpha} \xi_{j}^{\beta} \geq \lambda|\xi|^{2}, \quad \xi \in \mathbb{R}^{m N}
$$

and $f_{i}^{\alpha}, f^{\alpha}$ are $L^{2}$ functions defined in $\Omega$. In the following we will let $f=$ $\left(f^{1}, \ldots, f^{N}\right)$, and $f_{i}=\left(f_{i}^{1}, \ldots, f_{i}^{N}\right)$. Systems such as (1.6) are the most basic examples of non-diagonal systems of Hörmander type. In the context of the present paper they either arise as limits of the blow-up's of the Euler-Lagrange equation of (1.1), or as the "frozen" version of the homogeneous part of (1.3). We will prove that for smooth $f_{i}^{\alpha}$, and $f^{\alpha}$, the system (1.6) satisfies the following estimate: For each $n>0$ there exist $C_{n}>0$, and multi-indices $a, b, c, d$, depending on $n$, such that for any multi-index $k$ of order less than or equal to $n$

(1.8) $\sup _{B(0,1)}\left|X^{k} u\right| \leq C_{n}\left\{\int_{B(0,2)}|u|^{2} d x+\int_{B(0,2)}\left[\left|T^{a} X^{b} f^{\alpha}\right|^{2}+\left|T^{c} X^{d} f_{i}^{\alpha}\right|^{2}\right] d p\right\}$.

Here, $T$ denotes a derivative along the direction of commutators. As a consequence of (1.8) we find the following basic result. 
Theorem 1.3 Let $\mathbf{G}$ be a Carnot group of step two, then the system (1.6) is hypoelliptic in the sense that, given an open set $\Omega \subset \mathbf{G}$ and $f^{\alpha}, f_{i}^{\alpha} \in C^{\infty}(\Omega)$, a weak solution to (1.6) is $C^{\infty}(\Omega)$.

After one has the hypoellipticity result, then standard arguments lead to $L^{p}$, $1<p<\infty$, and Hölder estimates for higher order derivatives of the solutions.

It is important to note that for diagonal systems the $W^{2,2}$-estimates and the ensuing hypoellipticity follow from the fundamental works of J.J. Kohn [K], Hörmander $[\mathrm{H}]$, Folland and Stein [FS], and Folland [F]. In this connection, one should also see the interesting recent paper of Xu and Zuily [XZ]. However, the results in these papers do not cover non-diagonal systems and this is why we needed to develop a new proof of these basic results. After the completion of the present paper E. Stein, M. Christ and D. Geller brought to our attention that the $W^{2,2}$-estimates could also be derived from the analysis of pseudo-differential operators on homogeneous groups developed in [G], [CGGP], and [T]. Such approach is very general but also highly technical and complex. We feel that our proof, which is close in spirit to the works [C1], [C2], has perhaps the advantage of being more easily accessible to workers in pde's who are not familiar with the theory of pseudo-differential operators. Moreover, the method of fractional order difference quotients used here can be applied also to quasi-linear systems such as (1.3), or to those arising as Euler-Lagrange equations for (1.1). While for (1.3) the parametrix method can still be used (by freezing the coefficients), this is not the case for the Euler-Lagrange systems of (1.1), where in order to use the method of freezing the coefficients one needs to have a-priori regularity assumptions on the minimizers.

We use the method of fractional order difference quotients to prove the second part of Theorem 1.1. In order to estimate the size of the singular set one needs to establish higher differentiability of the minimizers not just in terms of the horizontal derivatives, but with respect to the underlying Riemannian structure. This is achieved by the following result.

Theorem 1.4 If $u$ is as in Theorem 1.1, with $q=2$, then its Riemannian gradient $\nabla u$ has locally square integrable horizontal derivatives.

In closing, we give a short description of the organization of the paper. Section two is devoted to some preliminary definitions and lemmas concerning Sobolev and Hölder spaces. We also collect various basic results concerning difference quotients. In section three we establish the $L^{2}$ estimates, the hypoellipticity for (1.6), and prove Theorem 1.4. In section four we present for the special case $f^{\alpha}=0$ the detailed blow-up analysis of (1.3), and we prove Theorem 1.1. In section five we prove Theorem 1.2.

Acknowledgements. We thank Michael Christ and Daryl Geller for interesting conversations regarding the method of the parametrix on homogeneous groups. We also thank the anonymous referee for carefully reading the manuscript, and for several comments that contributed to improve the presentation. 


\section{Preliminaries}

As we mentioned in the previous section this paper focuses on Carnot groups of step two. We will thus henceforth assume that $\mathbf{G}$ represents such a group with Lie algebra $\mathbf{g}=V^{1} \oplus V^{2}$, with $\left[V^{1}, V^{1}\right]=V^{2},\left[\mathbf{g}, V^{2}\right]=0$. We will denote by $m$ the dimension of $V^{1}$, and indicate with $k$ the dimension of $V^{2}$. The homogeneous dimension of $\mathbf{G}$ will be thus given by $Q=m+2 k$. Let $X_{1}, \ldots, X_{m}$ be an orthonormal basis of $V^{1}$ and fix an orthonormal basis $T_{1}, \ldots, T_{k}$ of $V^{2}$. The same symbols $X_{j}$ and $T_{l}$ will be used to indicate the corresponding left-invariant vector fields on $\mathbf{G}$. One can easily see that the Carnot Carathéodory metric - the control distance associated to the vector fields $X_{j}$ via Chow's accessibility theorem [Cho] - is equivalent to the pseudo-distance $d$. Since we will exclusively work with $d$, we will not make further reference to the control metric. If $B(p, r)$ denotes the pseudo-ball centered at $p$ with radius $r$, then as we said $|B(p, r)|=\omega_{\mathbf{G}} r^{Q}$.

In this paper we will always use the globally defined exponential coordinates on $\mathbf{G}$. It is possible to describe the group structure only in terms of the algebra by means of the formula of Baker-Campbell-Hausdorff (see Sect. 2.15 in [V]): If $X, Y \in \mathbf{g}$, then

$$
e^{X} e^{Y}=e^{X+Y+\frac{1}{2}[X, Y]}
$$

Using (2.1) one can write explicitly the dependence of the vector fields on the exponential coordinates. There exists constants $c_{i, j, l}, i, j=1, \ldots, m$, and $l=$ $1, \ldots, k$, such that if $(x, t) \in \mathbb{R}^{m} \oplus \mathbb{R}^{k}$ represent the exponential coordinates of $p \in \mathbf{G}$, then

$$
\begin{gathered}
X_{i}(p)=\frac{\partial}{\partial x_{i}}+\sum_{l=1}^{k} \sum_{j \neq i, j=1}^{m} c_{i, j, l} x_{j} \frac{\partial}{\partial t_{l}}, \quad i=1, \ldots, m, \\
T_{l}(p)=\frac{\partial}{\partial t_{l}}, \quad l=1, \ldots, k .
\end{gathered}
$$

We respectively denote by $X u=\left(X_{1} u, \ldots, X_{m} u\right)$ and $T u=\left(T_{1} u, \ldots, T_{k} u\right)$ the horizontal and non-horizontal gradient of a function $u$.

The simplest non-trivial example of Carnot group of step two is the Heisenberg group $\mathbb{H}^{n}$. The latter is the Lie group whose underlying manifold is $\mathbb{R}^{2 n} \times \mathbb{R}$ endowed with the non-commutative group law

$$
(z, t)(\zeta, s)=(z+\zeta, t+s+2[z, \zeta]),
$$

where $z, \zeta \in \mathbb{R}^{2 n}, t, s \in \mathbb{R}$, and $[z, \zeta]=\sum_{i=1}^{n}\left(\zeta_{i} z_{n+i}-z_{i} \zeta_{n+i}\right)$. The vector fields $X_{i}=\partial_{z_{i}}+2 z_{n+i} \partial_{t}, X_{i+n}=\partial_{z_{n+i}}-2 z_{i} \partial_{t}$, for $i=1, \ldots, n$, and $T=-4 \partial_{t}$ form a left-invariant basis for the Lie algebra of $\mathbb{H}^{n}$. The real part of the Kohn subLaplacian on $\mathbb{H}^{n}$ is $\mathcal{L}=\sum_{j=1}^{2 n} X_{j}^{2}$. The stratification of the Heisenberg algebra is given by $V^{1}=\operatorname{span}\left\{X_{1}, \ldots, X_{2 n}\right\}$, and $V^{2}=\operatorname{span}\{T\}$. The associated dilations are parabolic $\delta_{\lambda}(z, t)=\left(\lambda z, \lambda^{2} t\right)$, with homogeneous dimension $Q=2 n+2$. If $V$ is a left-invariant vector field and $V=\sum_{l=1}^{2 n} v_{l} X_{l}+v_{2 n+1} T$, then we will write the exponential map as $\exp (V)=e^{V}=\left(v_{1}, v_{2}, \ldots, v_{2 n+1}\right)=\left(v^{\prime}, v_{2 n+1}\right)$. 
In this case, the Baker-Campbell-Hausdorff formula follows immediately from the group law (2.2). Since the group is of step two the homogeneous gauge is given by $\|p\|=\left(|z|^{4}+t^{2}\right)^{1 / 4}$, where $p=(z, t) \in \mathbb{H}^{n}$, with relative pseudodistance $d\left(p, p^{\prime}\right)=\left\|p^{-1} p^{\prime}\right\|$. It was proved by Cygan [Cy] that this is in fact a distance.

Let $\mathbf{G}$ be a Carnot group of step two and $\Omega \subset \mathbf{G}$ an open subset. For $N \in \mathbb{N}$ consider functions $u: \Omega \rightarrow \mathbb{R}^{N}$. For $0<\alpha<1$ we will use the Folland-Stein class

$$
\Gamma^{\alpha}(\Omega)=\left\{u \in L^{\infty}(\Omega) \mid \sup _{p_{1}, p_{2} \in \Omega, p_{1} \neq p_{2}} \frac{\left|u\left(p_{1}\right)-u\left(p_{2}\right)\right|}{d\left(p_{1}, p_{2}\right)^{\alpha}}<\infty\right\},
$$

and its local version $\Gamma_{\text {loc }}^{\alpha}(\Omega)=\left\{u \mid \eta u \in \Gamma^{\alpha}(\Omega)\right.$ for every $\left.\eta \in C_{0}^{\infty}(\Omega)\right\}$, see [FS] and $[\mathrm{F}]$. For $k \in \mathbb{N}$ the symbol $\Gamma_{\text {loc }}^{k, \alpha}(\Omega)$ will denote the set of functions having horizontal derivatives up to order $k$ in $\Gamma_{\text {loc }}^{\alpha}(\Omega)$. Observe that $\Gamma^{\alpha}(\Omega)$ becomes a Banach space when endowed with the norm

$$
\|u\|_{\Gamma^{\alpha}(\Omega)}=\sup _{p_{1}, p_{2} \in \Omega, p_{1} \neq p_{2}} \frac{\left|u\left(p_{1}\right)-u\left(p_{2}\right)\right|}{d\left(p_{1}, p_{2}\right)^{\alpha}}+\|u\|_{L^{\infty}(\Omega)} .
$$

We will need the horizontal Sobolev spaces

$$
\begin{array}{r}
\mathcal{L}_{\mathrm{loc}}^{1, q}(\Omega)=\left\{f: \Omega \rightarrow \mathbb{R}^{N} \mid f \in L_{\mathrm{loc}}^{q}(\Omega), X f=\left(X_{1} f, \ldots, X_{m} f\right) \in L_{\mathrm{loc}}^{q}(\Omega)\right\}, \\
1 \leq q<\infty .
\end{array}
$$

Here, $X_{j} f, j=1, \ldots, m$ denotes the weak derivative of $f \in L_{\text {loc }}^{1}(\Omega)$ along $X_{j}$ defined by the formula

$$
\int_{\Omega} X_{j} f(p) \phi(p) d p=-\int_{\Omega} f(p) X_{j} \phi(p) d p, \quad \phi \in C_{o}^{\infty}(\Omega) .
$$

We recall that in any Carnot group one has $X_{j}^{*}=-X_{j}$ for the formal adjoint of $X_{j}$, see $[\mathrm{F}]$. We will also use the Sobolev space of functions with zero trace,

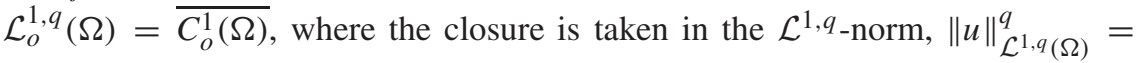
$\int_{\Omega}\left(|u|^{q}+|X u|^{q}\right) d x$. Let $h$ be an integer and denote by $I_{h}=\left(i_{1}, \ldots, i_{h}\right)$ a multiindex with $1 \leq i_{j} \leq m$. Given a function $f$ we indicate with $X^{I_{h}} f=X_{i_{1}} \ldots X_{i_{h}} f$ the corresponding horizontal derivative of order $h$. The higher order Sobolev spaces are

$$
\begin{aligned}
\mathcal{L}_{\mathrm{loc}}^{k, q}(\Omega)=\left\{f: \Omega \rightarrow \mathbb{R}^{N}\left|\sum_{I_{h}}\right| X^{I_{h}} f \mid \in L_{\mathrm{loc}}^{q}(\Omega), \text { for any } 0 \leq h \leq k\right\}, & \\
& 1 \leq q<\infty .
\end{aligned}
$$

We next recall that in a group of step two the gauge pseudo-balls are NTA domains, see [CG], hence, in particular, they are Poincaré-Sobolev (PS-) domains. Thereby, such sets support the Poincaré-Sobolev inequality, i.e., for every 
$1 \leq q<Q$ there exists $S_{q}>0$ such that for every $u \in S^{1, q}\left(B\left(p_{o}, r\right)\right)$ one has

$$
\begin{aligned}
& \left(\frac{1}{\left|B\left(p_{o}, r\right)\right|} \int_{B\left(p_{o}, r\right)}\left|u-u_{p_{o}, r}\right|^{\frac{Q q}{Q-q}} d x\right)^{\frac{Q-q}{Q q}} \\
& \quad \leq S_{q} r\left(\frac{1}{\left|B\left(p_{o}, r\right)\right|} \int_{B\left(p_{o}, r\right)}|X u|^{q} d x\right)^{\frac{1}{q}},
\end{aligned}
$$

where we have let $u_{p_{o}, r}$ denote the average of $u$ over the pseudo-ball $B\left(p_{o}, r\right)$. See, e.g., $[\mathrm{Lu}],[\mathrm{GN}]$ or also $[\mathrm{HK}]$. The fact that gauge pseudo-balls are (PS)-domains allows to use Theorem 1.27 in $[\mathrm{GN}]$ and obtain the following compact embedding

$$
\mathcal{L}^{1, q}\left(B\left(p_{o}, r\right)\right) \hookrightarrow \hookrightarrow L^{q}\left(B\left(p_{o}, r\right)\right), \quad 1 \leq q<\infty .
$$

We will need the analogue of the Morrey-Campanato embedding, proved by Folland (see Theorem $5.15[\mathrm{~F}]$ ): For every $p_{o} \in \mathbf{G}, k \in \mathbb{N}, 1 \leq q<\infty$, and $r>0$, one has

$$
\mathcal{L}^{k, q}\left(B\left(p_{o}, r\right)\right) \subset \Gamma_{\text {loc }}^{\alpha}\left(B\left(p_{o}, r\right)\right)
$$

where $\alpha=k-\frac{Q}{q}$.

The spaces $\mathcal{L}_{\text {loc }}^{1, q}(\Omega)$, with $q<\infty$, can be characterized in terms of difference quotients. Let $Z$ be a smooth vector field in $\Omega$. A function $f \in L_{\text {loc }}^{1}(\Omega)$ is weakly differentiable in $\Omega$ along the direction $Z$, if there exists a locally integrable function $g$ such that for any $\phi \in C_{o}^{\infty}(\Omega)$ the identity

$$
\int_{\Omega} f(p) Z^{*} \phi(p) d p=\int_{\Omega} g(p) \phi(p) d p
$$

holds. Here $Z^{*}$ denotes the formal adjoint of $Z$. For $0<s<\varepsilon$, where $\varepsilon$ is suitably small, denote with $p e^{s Z}$ the integral curve of $Z$ starting at $p$. If $K \subset \subset \Omega$ is an open set, then we can think of $e^{s Z}$ as a diffeomorphism $e^{s Z}: K \rightarrow e^{s Z}(K) \subset \Omega$. We also let $e^{s Z} f(p)=f\left(p e^{s Z}\right)$ when $p \in K$ and $f$ is a real valued function defined on $e^{s Z}(K)$.

Proposition 2.1 Let $u \in L_{\mathrm{loc}}^{q}(\Omega), 1 \leq q<\infty$, and $K \subset \subset \Omega$. If there exist positive constants $\varepsilon, C$ such that

$$
\sup _{0<|s|<\varepsilon} \int_{K} \frac{\left|u\left(x e^{s Z}\right)-u(x)\right|^{q}}{|s|^{q}} d x \leq C^{q},
$$

then $Z u \in L^{q}(K)$ and $\|Z u\|_{L^{q}(K)} \leq C$. On the other hand, if $Z u \in L_{\mathrm{loc}}^{q}(\Omega)$, then (2.3) holds with $C=2\|Z u\|_{L^{q}(K)}$ for any $K \subset \subset \Omega$.

Proof. The proof of this proposition is standard, see for instance Proposition 2.3 in $[\mathrm{C} 1]$. 
In the following we will need estimates on the Lebesgue norm of fractional derivatives of functions in the direction of commutators $T$. Let $\Omega, Z$ be as as in Proposition 2.1. For $w \in L^{2}(\Omega)$ with compact support in $\Omega$, and $\alpha \in(0,1)$, we define the seminorm

$$
|w|_{Z, \alpha}^{2}=\sup _{|h|<\varepsilon_{0}} \int_{\Omega}|h|^{-2 \alpha}\left|w\left(q e^{h Z}\right)-w(q)\right|^{2} d q,
$$

where $\varepsilon_{O}$ is chosen sufficiently small. Let $n=m+k=\operatorname{dim}(\mathbf{g})$, and identify $\mathbf{g}$ with the Euclidean space $\mathbb{R}^{n}$. Now consider a function $w \in C_{o}^{\infty}\left(\mathbb{R}^{n}\right)$. For $\alpha \in(0,1)$ the fractional derivative of $w$ of order $\alpha$, along the direction $e_{j}, j=1, \ldots, n$, is given by

$$
\frac{\widehat{\partial^{\alpha} w}}{\partial e_{j}}=|h|^{\alpha} \widehat{w}\left(x_{1}, . ., x_{j-1}, h, x_{j+1}, \ldots, x_{n}\right),
$$

where we have denoted by $\hat{w}$ the partial Fourier transform in the variable $e_{j}$. By the Plancherel formula we have

$$
\left.\left\|\frac{\partial^{\alpha} w}{\partial e_{j}}\right\|_{L^{2}(\mathbf{g})}=\int_{\mathbb{R}^{n}}|h|^{2 \alpha} \mid \hat{w}\left(x_{1}, . ., x_{j-1}, h, x_{j+1}, \ldots, x_{n}\right)\right)\left.\right|^{2} d x d h,
$$

The following theorem of Peetre [P] (see also sec. 2.3.3, Remark 4, p. 180 in [Tr]) will play an important role in the sequel.

Theorem 2.2 Let $0<\beta<\alpha<1$, and $w \in C_{o}^{\infty}\left(\mathbb{R}^{n}\right)$. There exists a positive constant $C$, depending only on $\alpha$ and $\beta$, such that for each vector $e_{j}, j=1, \ldots, n$, of the standard basis of $\mathbb{R}^{n}$, one has

$$
C\left\|\frac{\partial^{\beta} w}{\partial e_{j}}\right\|_{L^{2}\left(\mathbb{R}^{n}\right)} \leq|w|_{\frac{\partial}{\partial e_{j}}, \alpha} \leq C^{-1}\left\|\frac{\partial^{\alpha} w}{\partial e_{j}}\right\|_{L^{2}\left(\mathbb{R}^{n}\right)} .
$$

The next result, which is a direct consequence of the formula of BakerCampbell-Hausdorff, is a special case of Theorem 4.3 in Hörmander's fundamental paper $[\mathrm{H}]$.

Theorem 2.3 Let $\mathbf{G}$ be a Carnot group of step two, let $\Omega \subset \mathbf{G}$ be an open set and $B\left(p_{o}, r\right) \subset \Omega$ a gauge pseudo-ball. If $\eta \in C_{o}^{\infty}\left(B\left(p_{o}, r\right)\right)$, and $w \in C^{\infty}(\Omega)$, then there exists a positive constant $C$ such that for each $T \in V^{2}$.

$$
|w \eta|_{T, \frac{1}{2}} \leq C \sum_{l=1}^{2 n}|w \eta|_{X_{l}, 1}
$$

At this point we turn our attention to weak solutions to (1.6). To state our next result we introduce a definition. For a given left-invariant vector field $Z$ and a function $f: \mathbf{G} \rightarrow \mathbb{R}$ we let for $s \neq 0$ fixed

$$
(f)_{Z, \gamma}(p)=\frac{f\left(p e^{s Z}\right)-f(p)}{|s|^{\gamma}} .
$$


Theorem 2.4 Let $\mathbf{G}$ be a Carnot group of step two and $\Omega \subset \mathbf{G}$ be an open set. If $u \in \mathcal{L}_{\text {loc }}^{1,2}(\Omega)$ is a weak solution of (1.6) in $\Omega$, then for every left-invariant vector field $Z$, the function $(u)_{Z, \gamma}=\left(\left(u^{1}\right)_{Z, \gamma}, \ldots,\left(u^{N}\right)_{Z, \gamma}\right)$ satisfies the following system

$$
\begin{aligned}
& \sum_{\beta=1}^{N} \sum_{i, j=1}^{m} \int_{\Omega}\left[A_{i j}^{\alpha \beta}\left(X_{j} u^{\beta}\right)_{Z, \gamma}(p)+\left(f_{i}^{\alpha}\right)_{Z, \gamma}(p)\right] X_{i} \phi^{\alpha}(p) d p \\
& +\sum_{\beta=1}^{N} \sum_{i, j=1}^{m} \int_{\Omega}\left[A_{i j}^{\alpha \beta} X_{j} u^{\beta}\left(p e^{s Z}\right)+f_{i}^{\alpha}\left(p e^{s Z}\right)\right] \frac{s|s|^{-\gamma}}{2}\left[Z, X_{i}\right] \phi^{\alpha}(p) d p \\
& =\int_{\Omega} f^{\alpha}(p)\left(\phi^{\alpha}\right)_{-Z, \gamma}(p) d p, \quad \alpha=1, \ldots, N,
\end{aligned}
$$

for every $\phi=\left(\phi^{1}, \ldots, \phi^{N}\right) \in C_{o}^{\infty}\left(\Omega, \mathbb{R}^{N}\right), \gamma \in(0,1)$, and $|s|$ small enough.

Proof. The proof is based on the Baker-Campbell-Hausdorff formula. The first step consists in writing the weak form of (1.6)

$$
\sum_{\beta=1}^{N} \sum_{i, j=1}^{m} \int_{\Omega}\left(A_{i j}^{\alpha \beta} X_{j} u^{\beta}+f_{i}^{\alpha}\right)(p) X_{i} \phi^{\alpha}(p) d p=\int_{\Omega} f^{\alpha}(p) \phi^{\alpha}(p) d p
$$

where $\phi=\left(\phi^{1}, \ldots, \phi^{N}\right) \in C_{o}^{\infty}\left(\Omega, \mathbb{R}^{N}\right)$. We also write a similar equation with test function $\tilde{\phi}(p)=\phi\left(p e^{-s Z}\right)$, subtract one of the other, divide by $|s|^{\gamma}$ and change variable $p \rightarrow p e^{s Z}$, to obtain

$$
\begin{aligned}
& \sum_{\beta=1}^{N} \sum_{i, j=1}^{m} \int_{\Omega}\left[A_{i j}^{\alpha \beta}\left(X_{j} u^{\beta}\right)_{Z, \gamma}(p)+\left(f_{i}^{\alpha}\right)_{Z, \gamma}(p)\right] X_{i} \phi^{\alpha}(p) d p \\
& +\sum_{\beta=1}^{N} \sum_{i, j=1}^{m} \int_{\Omega}\left[A_{i j}^{\alpha \beta} X_{j} u^{\beta}\left(p e^{s Z}\right)+f_{i}^{\alpha}\left(p e^{s Z}\right)\right] \\
& \times \frac{X_{i}\left[\phi^{\alpha}\left(p e^{-s Z}\right)\right]\left(p e^{s Z}\right)-X_{i} \phi^{\alpha}(p)}{|s|^{\gamma}} d p \\
& =\int_{\Omega} f^{\alpha}(p)\left(\phi^{\alpha}\right)_{-Z, \gamma}(p) d p,
\end{aligned}
$$

(recall that the Jacobian of the translation $p \rightarrow p e^{s Z}$ is the constant function with value one, see for instance [CoG]). The Baker-Campbell-Hausdorff formula yields

$$
\frac{X_{i}\left[\phi^{\alpha}\left(p e^{-s Z}\right)\right]\left(p e^{s Z}\right)-X_{i} \phi^{\alpha}(p)}{|s|^{\gamma}}=\frac{s|s|^{-\gamma}}{2}\left[Z, X_{i}\right] \phi^{\alpha}(p),
$$

concluding the proof.

In several occasions we will make use of a basic energy estimate, which in the standard elliptic case is usually referred to as Caccioppoli inequality. In the next lemma we will state such estimate for weak solutions of the system (1.3) (of which 
the constant coefficient system (1.6) is a special case), but it should be remarked here that, as in the classical setting (see for instance [Gi]), it holds in much greater generality.

Lemma 2.5 Let $\mathbf{G}$ be a Carnot group of step two, and let $\Omega \subset \mathbf{G}$ be a bounded open set. If $u \in \mathcal{L}_{\mathrm{loc}}^{1,2}(\Omega)$ is a weak solution of (1.3) in $\Omega$, with the assumptions (1.4), (1.5) and with $f^{\alpha}=0, \alpha=1, \ldots, N$, then the following Caccioppoli type inequality holds for $B\left(p_{o}, 2 r\right) \subset \Omega$

$$
\int_{B\left(p_{o}, r\right)}|X u|^{2} d p \leq C \frac{1}{r^{2}} \int_{B\left(p_{o}, 2 r\right)}|u|^{2} d p .
$$

Proof. The proof follows along the lines of the classical argument, see, e.g., [Gi]. One multiplies both sides of (1.3) by $u \eta^{2}$, where $\eta \in C_{o}^{\infty}\left(B\left(p_{o}, 2 r\right)\right), \eta \geq 0$, $\eta \equiv 1$ in $B\left(p_{o}, r\right)$, and $|X \eta| \leq C / r$. After integrating on $B\left(p_{o}, 2 r\right)$, and using Hölder inequality and the coercivity assumptions (1.4), the conclusion follows. The existence of test functions $\eta$ with the properties described above can be easily established using the group translations and the pseudo-norm $\|\cdot\|$.

\section{Systems with constant coefficients}

In this section we prove the hypoellipticity of the constant coefficient system (1.6)

$$
-\sum_{\beta=1}^{N} \sum_{i, j=1}^{m} X_{i}\left(A_{i j}^{\alpha \beta} X_{j} u^{\beta}+f_{i}^{\alpha}\right)=f^{\alpha}, \alpha=1, \ldots, N
$$

Our approach is based on appropriate sub-elliptic versions, contained in Theorems 3.5 and 3.6, of the classical $W^{2,2}$ estimates. Following the classical scheme we differentiate the system with respect to a vector field $Z$, and show that the function $Z u$ solves a new system, similar in structure to (1.6). A major obstacle is constituted by the non-trivial commutation relations involving the horizontal basis $X=\left\{X_{1}, \ldots, X_{m}\right\}$. In fact, when (1.6) is differentiated new terms arise which include derivatives of $u$ along the directions of the commutators $\left[X_{i}, Z\right]$. Since a-priori there is no control on such terms, it is necessary to develop new ideas. In essence, we combine in a delicate way a repeated use of the fractional difference quotients along the integral curves of $Z$, with the energy inequality in Lemma 2.5, and the interpolation estimates in Theorems 2.2 and 2.3. In the end, the iteration of the sub-elliptic $W^{2,2}$ estimates allows to establish the smoothness of weak solutions.

To conclude the section, in Theorem 3.9 we will prove a priori estimates for quasi-linear systems with quadratic growth in the horizontal gradient. Such estimates, which are derived by the same technique employed in Theorems 3.1 and 3.2 , will be used in the proof of Theorem 4.11. Henceforth, we use the notation $f_{i}=\left(f_{i}^{1}, \ldots, f_{i}^{N}\right), i=1, \ldots, m, f=\left(f^{1}, \ldots, f^{N}\right)$. 
Theorem 3.1 Let $\mathbf{G}$ be a Carnot group of step two, and $u \in \mathcal{L}_{\mathrm{loc}}^{1,2}(\Omega)$ be a weak solution to (1.6) in an open set $\Omega \subset \mathbf{G}$. If $f^{\alpha}, T_{j} f^{\alpha}, f_{i}^{\alpha}, T_{j} f_{i}^{\alpha} \in L_{\mathrm{loc}}^{2}(\Omega)$, for every $i=1, \ldots, m, j=1, \ldots, k$, and $\alpha=1, \ldots, N$, then for each $T_{j_{o}} \in V^{2}$ one has

$$
T_{j_{o}} u \in \mathcal{L}_{\mathrm{loc}}^{1,2}(\Omega)
$$

Furthermore, for every pseudo-ball $B\left(p_{o}, 2 r\right) \subset \Omega$ and $\eta \in C_{o}^{\infty}\left(B\left(p_{o}, 2 r\right)\right)$, the following estimate holds for $w=\eta T_{j_{o}} u$

$$
\begin{gathered}
\int_{B\left(p_{o}, r\right)}\left(r^{4}|w|^{2}+r^{6}|X w|^{2}\right) d p \\
\leq C \int_{B\left(p_{o}, 2 r\right)}\left(r^{2}|X u|^{2}+|u|^{2}+r^{4}|f|^{2}+r^{8}\left|T_{j_{o}} f\right|^{2}\right. \\
\left.+\sum_{i=1}^{m}\left[r^{2}\left|f_{i}\right|^{2}+r^{6}\left|T_{j_{o}} f_{i}\right|^{2}\right]\right) d p,
\end{gathered}
$$

for some positive constant $C$ depending only on $\mathbf{g}$ and on the structural parameter in (1.7). Moreover, $T_{j_{o}}$ u is a weak solution in $\Omega$ to

$$
-\sum_{\beta=1}^{N} \sum_{i, j=1}^{m} X_{i}\left(A_{i, j}^{\alpha \beta} X_{j}\left(T_{j_{o}} u\right)^{\beta}+T_{j_{o}} f_{i}^{\alpha}\right)=T_{j_{o}} f^{\alpha}
$$

for $\alpha=1, \ldots, N$.

Proof. Since the class of systems satisfying (1.7) is invariant under the action of the group left-translations it is enough to show that $w \in \mathcal{L}_{\text {loc }}^{1,2}(B(e, 4 r))$, where $e$ is the group identity and $B(e, 4 r) \subset \Omega$. By dilation invariance we can assume $r=1$. Once (ii) has been proved for $r=1$, the general case follows by rescaling. We use difference quotients in the $T_{j_{o}}$ direction of order (roughly) one half. For $0<\gamma<1$ define

$$
u_{\left(T_{j_{o}}, \gamma\right)}^{\beta}(p)=|\sigma|^{-2 \gamma}\left(u^{\beta}\left(p e^{\sigma^{2} T_{j_{o}}}\right)-u^{\beta}(p)\right) .
$$

The differentiated system (2.6) takes a simpler form in the case $Z=T_{j_{o}}$ and $\gamma=1 / 2$. For every $\phi \in C_{o}^{\infty}\left(\Omega, \mathbb{R}^{N}\right)$ one has

$$
\sum_{\beta=1}^{N} \sum_{i, j=1}^{m} \int_{\Omega}\left[A_{i j}^{\alpha \beta}\left(X_{j} u^{\beta}\right)_{\left(T_{j_{o}}, 1 / 2\right)}+\left(f_{i}^{\alpha}\right)_{\left(T_{j_{o}}, 1 / 2\right)}\right] X_{i} \phi^{\alpha} d p=\int_{\Omega} f^{\alpha} \phi_{\left(-T_{j_{o}}, 1 / 2\right)}^{\alpha} d p .
$$

Now observe that (3.1) continues to hold for test functions in $\mathcal{L}_{o}^{1,2}\left(\Omega, \mathbb{R}^{N}\right)$. Since $T_{j_{o}}$ commutes with the $X_{j}$ 's, then the function $p \rightarrow u\left(p e^{\sigma^{2} T_{j_{o}}}\right)$ is in $\mathcal{L}^{1,2}(B(e, 4))$. If we set $\phi^{\alpha}(p)=\left(u^{\alpha}\right)_{\left(T_{j_{o}}, 1 / 2\right)}(p) \eta^{2}(p)$, with $\eta$ as in the statement of the theorem, 
then $\phi$ is a good test function for (3.1). From the coercivity hypothesis, and with this choice of test function, we obtain from (3.1) the following energy inequality

$$
\begin{gathered}
\int_{\Omega}\left|\left(X u^{\alpha}\right)_{\left(T_{j_{o}}, 1 / 2\right)}\right|^{2} \eta^{2} d p \\
\leq C \int_{\Omega}\left[\left|\left(u^{\alpha}\right)_{\left(T_{j_{o}}, 1 / 2\right)}\right|^{2}\left(\eta^{2}+|X \eta|^{2}\right)\right. \\
\left.+\sum_{i=1}^{m}\left|\left(f_{i}\right)_{\left(T_{j_{o}}, 1 / 2\right)}\right|^{2} \eta^{2}+\left|(f)_{\left(T_{j_{o}}, 1 / 2\right)}\right|^{2} \eta\right] d p .
\end{gathered}
$$

Noting that $\left|\eta_{\left(T_{j_{o}}, 1 / 2\right)}\right| \leq C$, then (3.2) and Theorems 2.2 and 2.3 yield

$$
\int_{\Omega}\left|\partial_{t_{j_{o}}}^{\frac{1}{2}-\varepsilon} X\left(\eta u^{\alpha}\right)\right|^{2} d p
$$

$$
\leq C \int_{B(e, 2)}\left(|u|^{2}+|X u|^{2}+\sum_{i=1}^{m}\left[\left|f_{i}\right|^{2}+\left|T_{j_{o}} f_{i}\right|^{2}\right]+|f|^{2}+\left|T_{j_{o}} f\right|^{2}\right) d p .
$$

To improve in the order of differentiability of $u$ we use Theorem 2.3 once more

$$
\begin{gathered}
\int_{\Omega}\left|\partial_{t_{j o}}^{1-2 \varepsilon} \eta u^{\alpha}\right|^{2} d p \\
\leq C \int_{B(e, 2)}\left(|u|^{2}+|X u|^{2}+\sum_{i=1}^{m}\left[\left|f_{i}\right|^{2}+\left|T_{j_{o}} f_{i}\right|^{2}\right]+|f|^{2}+\left|T_{j_{o}} f\right|^{2}\right) d p .
\end{gathered}
$$

We now apply (3.4) with $\varepsilon_{o}=1 / 12$ to gain control over the $L^{2}$ norm of $\partial_{t_{j_{o}}}^{\frac{5}{6}} u^{\alpha}$. Next, we consider the difference quotients $u_{\left(T_{j_{o}}, 5 / 6\right)}$, repeat the argument in (3.1)-(3.3), and use (3.4) to obtain

$$
\begin{gathered}
\int_{\Omega}\left(\left|\partial_{t_{j_{o}}}^{\frac{5}{6}-\varepsilon}\left(\eta u^{\alpha}\right)\right|^{2}+\left|X \partial_{t_{j_{o}}}^{\frac{5}{6}-\varepsilon}\left(\eta u^{\alpha}\right)\right|^{2}\right) d p \\
\leq C \int_{B(e, 2)}\left(|u|^{2}+|X u|^{2}+\sum_{i=1}^{m}\left[\left|f_{i}\right|^{2}+\left|T_{j_{o}} f_{i}\right|^{2}\right]+|f|^{2}+\left|T_{j_{o}} f\right|^{2}\right) d p .
\end{gathered}
$$

At this point we are ready to estimate

$$
\begin{aligned}
& \int_{\Omega}\left|T\left(\eta u^{\alpha}\right)\right|^{2} d p \leq \int_{\Omega}\left|\partial_{t_{j_{o}}}^{\frac{1}{2}-\varepsilon_{o}} \partial_{t_{j_{o}}}^{\frac{5}{6}-\varepsilon_{o}}\left(\eta u^{\alpha}\right)\right|^{2} d p \leq \int_{\Omega}\left|X \partial_{t_{j_{o}}}^{\frac{5}{6}-\varepsilon_{o}}\left(\eta u^{\alpha}\right)\right|^{2} d p \\
& \leq \int_{B(e, 2)}\left(|u|^{2}+|X u|^{2}+\sum_{i=1}^{m}\left[\left|f_{i}\right|^{2}+\left|T_{j_{o}} f_{i}\right|^{2}\right]+|f|^{2}+\left|T_{j_{o}} f\right|^{2}\right) d p .
\end{aligned}
$$

This proves the first part of the theorem. To establish (ii) it now suffices to consider the following difference quotients of order one: $\left(u^{\alpha}\right)_{\left(T_{j_{0}}, 1\right)}$, and apply Lemma 2.5 to (2.6) for this special case. Part (iii) follows from part (i) and from (2.6). 
In the sequel for a function $f$ defined in $\mathbf{G}$ we indicate with $\nabla f$ the standard Riemannian gradient of $f$, whereas we let $|T f|^{2}=\left(\sum_{l=1}^{k}\left(T_{l} f\right)^{2}\right)$.

Theorem 3.2 Let $\mathbf{G}, u$, and $\Omega$ be as in Theorem 3.1. If $f^{\alpha}, T_{j} f^{\alpha} \in L_{\text {loc }}^{2}(\Omega)$ and $f_{i}^{\alpha}, \nabla f_{i}^{\alpha} \in L_{\mathrm{loc}}^{2}(\Omega)$, for $i=1, \ldots, m, j=1, \ldots, k$ and $\alpha=1, \ldots, N$, then for every $i_{o}=1, \ldots, m$ one has

$$
X_{i_{o}} u \in \mathcal{L}_{\mathrm{loc}}^{1,2}(\Omega)
$$

Moreover, for any pseudo-ball $B\left(p_{o}, 2 r\right) \subset \Omega$ and $\eta \in C_{o}^{\infty}\left(B\left(p_{o}, 2 r\right)\right)$ one has for $v=\eta X_{i_{o}} u$

$$
\int_{B\left(p_{o}, r\right)} r^{4}|X v|^{2} d p
$$

$$
\begin{aligned}
& \leq C \int_{B\left(p_{o}, 2 r\right)}\left(r^{2}|X u|^{2}+|u|^{2}\right. \\
& \left.\quad+\sum_{i=1}^{m}\left[r^{2}\left|f_{i}\right|^{2}+r^{4}\left|X f_{i}\right|^{2}+r^{6}\left|T f_{i}\right|^{2}\right]+r^{4}|f|^{2}+r^{8}|T f|^{2}\right) d p,
\end{aligned}
$$

for some positive constant $C$ depending only on $\mathbf{G}$ and the structural assumptions in (1.6). Finally, if we also assume that $f^{\alpha} \in \mathcal{L}_{\mathrm{loc}}^{1,2}(\Omega)$, then $X_{i_{o}}$ is a weak solution in $\Omega$ to

$$
\begin{gathered}
\sum_{\beta=1}^{N} \sum_{i, j=1}^{m} X_{i}\left(A_{i, j}^{\alpha \beta} X_{j} X_{i_{o}} u^{\beta}+A_{i, j}^{\alpha \beta}\left[X_{i_{o}}, X_{j}\right] u^{\beta}+X_{i_{o}} f_{i}^{\alpha}\right) \\
+\sum_{\beta=1}^{N} \sum_{i, j=1}^{m}\left[X_{i_{o}}, X_{i}\right]\left(A_{i j}^{\alpha \beta} X_{j} u^{\beta}+f_{i}^{\alpha}\right)=X_{i_{o}} f^{\alpha}, \quad \alpha=1, \ldots, N .
\end{gathered}
$$

We mention explicitly that an important consequence of Theorems 3.1 and 3.2 is that

$$
X_{i} u \in W_{\mathrm{loc}}^{1,2}(\Omega)
$$

where $W_{\text {loc }}^{1,2}(\Omega)$ denotes the ordinary Sobolev space.

Before proving Theorem 3.2 we need a preliminary lemma.

Lemma 3.3 Let $\mathbf{G}$ be a Carnot group of step two and $\Omega \subset \mathbf{G}$ be an open set. If $f \in \mathcal{L}_{\mathrm{loc}}^{1,2}(\Omega)$ is such that $T_{j} f \in L_{\mathrm{loc}}^{2}(\Omega)$, for $j=1, \ldots, k$, then for each $\theta \in \mathbf{G}$, the function $g(p)=f(p \theta)$ is in $\mathcal{L}_{\mathrm{loc}}^{1,2}(\tilde{\Omega})$, where $\tilde{\Omega}=\Omega \theta^{-1}$. Moreover, one has the identity:

$$
X_{i}\left(f\left(p e^{S}\right)\right)=X_{i} f\left(p e^{S}\right)-\left[S, X_{i}\right] f\left(p e^{S}\right),
$$

where without loss of generality we have set $\theta=e^{S}$. 
Proof. In the Heisenberg group the proof is a simple application of the BakerCampbell-Hausdorff formula. For the general case the argument is slightly different and is based on the following identity (see Chap. 1 in [CoG], or Sect. 2.5 in [V]): For $X, Y \in g$

$$
(\text { Ad } \exp X) Y=\sum_{k=0}^{\infty} \frac{1}{k !}(\operatorname{ad} X)^{k} Y=e^{\operatorname{ad} X} Y,
$$

where, if $P \in \mathbf{G}$, then Ad $P: g \rightarrow g$ is the differential of the map $Q \rightarrow P Q P^{-1}$, and $(\operatorname{ad} X) Y=[X, Y]$. In a nilpotent Lie group the infinite sum will simply be a finite sum, the highest order of non-zero commutators being the step of the group (in our setting hence, a sum of two terms).

At this point we observe that

$$
\begin{gathered}
X_{i}\left(f\left(p e^{S}\right)\right)=\left.\frac{d}{d t} f\left(p e^{t X_{i}} e^{S}\right)\right|_{t=0}=\left.\frac{d}{d t} f\left(p e^{S}\left(e^{-S}\right) e^{t X_{i}} e^{S}\right)\right|_{t=0} \\
=\left(\left(\left(\operatorname{Ad} e^{-S}\right) X_{i}\right) f\right)\left(p e^{S}\right)=\left(\sum_{k=0}^{\text {Step of } \mathbf{G}}(-1)^{k} \frac{(\operatorname{ad} S)^{k} X_{i}}{k !} f\right)\left(p e^{S}\right) .
\end{gathered}
$$

In our case, with a group of step two the formula becomes

$$
X_{i}\left(f\left(p e^{S}\right)\right)=X_{i} f\left(p e^{S}\right)-\left[S, X_{i}\right] f\left(p e^{S}\right) .
$$

The proof is concluded once we observe that $\left[S, X_{i}\right] \in V^{2}$ and the vectors $T_{k}$ are a basis of $V^{2}$.

Thanks to Theorem 3.1 (i), Lemma 3.3 allows to use difference quotients in the direction of $X_{i}, i=1, \ldots, m$.

Remark 3.4 Using the argument in Lemma 3.3 one can easily prove

$$
X_{i}\left(u_{\left(X_{i_{o}}, 1\right)}\right)=\left(X_{i} u\right)_{\left(X_{i_{0}}, 1\right)}+\left[X_{i}, X_{i_{0}}\right] u\left(p e^{\sigma X_{i_{0}}}\right) .
$$

In particular, this shows that if $u \in W_{\mathrm{loc}}^{1,2}(\Omega)$, then $u_{\left(X_{i_{0}}, 1\right)} \in \mathcal{L}_{\mathrm{loc}}^{1,2}(\Omega)$.

Proof of Theorem 3.2. The proof is based on Theorem 3.1. In particular, what makes things work is the fact that the solution $u$ is not only in the horizontal Sobolev space $\mathcal{L}_{\text {loc }}^{1,2}(\Omega)$, but also in the usual Euclidean Sobolev space $W_{\text {loc }}^{1,2}(\Omega)$. This follows from Theorem 3.1, which asserts the $L^{2}$-integrability of the derivatives in the directions of the second layer $V^{2}$, and from the representation of the vector fields in exponential coordinates (see section two).

As in the proof of Theorem 3.1, it suffices to establish (ii) in a fixed pseudoball $B(e, 1)$, and then use left-translations and dilations to obtain (ii) for a general pseudo-ball $B\left(p_{o}, r\right)$. Observe that by continuity (2.6) still holds for any $\phi$ in the ordinary Sobolev space $W_{o}^{1,2}\left(\Omega, \mathbb{R}^{N}\right)$. Let $\eta$ be as in the statement of the theorem. By Remark 3.4 we may use $\phi=u_{\left(X_{i_{0}}, 1\right)} \eta^{2}$ as a test function in (2.6), thus we 
proceed to substitute it in the equation. By Remark 3.4 and the coercivity condition (1.7) we derive the energy inequality

$$
\int_{\Omega}\left|\left(X \eta u^{\alpha}\right)_{\left(X_{i_{0}}, 1\right)}\right|^{2} d p \leq
$$

$C \int_{B(e, 2)}\left(|u|^{2}+|X u|^{2}+\left|T(u)_{\left(X_{i_{0}}, 1\right)}\right|^{2}\right.$

$$
\left.+\sum_{i=1}^{m}\left[\left|X f_{i}\right|^{2}+\left|f_{i}\right|^{2}\right]+\left|f^{\alpha}\right|\left|\left(\left(u^{\alpha}\right)_{\left(X_{i_{0}}, 1\right)} \eta^{2}\right)_{\left(X_{i_{0}}, 1\right)}\right|\right) d p .
$$

The conclusion now follows using Theorem 3.1 and Proposition 2.1.

When studying the regularity theory of weak solutions to the quasi-linear system (1.3) one is naturally led to analyze the hypoellipticity of linear sub-elliptic systems with constant coefficients of the type (1.6). In doing this one naturally encounters systems such as (2.6). For $A_{i j}^{\alpha \beta}$ as in (1.7) and $f^{\alpha}$ a smooth function we thus consider weak solutions of

$$
\sum_{\beta=1}^{N} \sum_{i, j=1}^{m} A_{i j}^{\alpha \beta} X_{i} X_{j} u^{\beta}=f^{\alpha},
$$

for $\alpha=1, \ldots, N$. By iterating Theorems 3.1 and 3.2 we obtain the following.

Theorem 3.5 Let $\mathbf{G}$ be a Carnot group of step two and $\Omega \subset \mathbf{G}$ be open. Let $u \in \mathcal{L}_{\text {loc }}^{1,2}(\Omega)$ be a weak solution of (3.7) in $\Omega$. For each $l, h \in \mathbb{N}$ and multi-indices $I_{h}=\left(i_{1}, \ldots, i_{h}\right) \in\{1,2, . ., m\}^{h}$, and $J_{l}=\left(j_{1}, \ldots, j_{l}\right) \in\{1,2, \ldots, k\}^{l}$, define

$$
v_{l, h}^{\beta}=T^{J_{l}} X^{I_{h}} u^{\beta}=\left(T_{j_{1}} \ldots T_{j_{l}}\right)\left(X_{i_{1}} \ldots X_{i_{h}}\right) u^{\beta} .
$$

If $f=\left(f^{1}, \ldots, f^{N}\right) \in C^{\infty}\left(\Omega, \mathbb{R}^{N}\right)$ then

$$
v_{l, h} \in \mathcal{L}_{\mathrm{loc}}^{1,2}(\Omega) .
$$

Moreover, $v_{l, h}$ is a solution to the system

$$
\sum_{\beta=1}^{N} \sum_{i, j=1}^{m} X_{i}\left(A_{i j}^{\alpha \beta} X_{j} v_{l, h}+f_{i,(l, h)}^{\alpha}\right)=f_{(l, h)}^{\alpha}, \quad \alpha=1, \ldots, N,
$$

where $f_{(l, 0)}^{\alpha}=T^{J_{l}} f^{\alpha}, f_{i,(l, 0)}^{\alpha}=0$,

$$
f_{i,(l, h)}^{\alpha}=T_{j_{l}} X_{i_{h}} f_{i,(l-1, h-1)}^{\alpha}+A_{i j}^{\alpha \beta}\left[X_{i_{h}}, X_{j}\right] T_{j_{l}} v_{l-1, h-1}^{\beta},
$$

and

$$
f_{l, h}^{\alpha}=T_{j_{l}}\left\{\left[X_{i_{h}}, X_{i}\right]\left(A_{i j}^{\alpha \beta} X_{j} v_{(l-1, h-1)}^{\beta}+f_{i,(l-1, h-1)}^{\alpha}\right)+X_{i_{h}} f_{(l-1, h-1)}^{\alpha}\right\} .
$$


We define the semi-norm $\|g\|_{l, h, R}=\sum_{I_{h}, J_{l}}\left\|T^{J_{l}} X^{I_{h}} g\right\|_{L^{2}(B(p, r)}$, where $I_{h}$ and $J_{l}$ respectively range over all the elements of $\{1,2, \ldots, m\}^{h}$ and of $\{1,2, \ldots, k\}^{l}$. As a consequence of Theorems 3.1, 3.2 and 3.5 one obtains the following.

Theorem 3.6 Let $\mathbf{G}, \Omega, B\left(p_{o}, r\right)$, and $u$ be as in the previous theorem. For each $h, l \in \mathbb{N}$, one has

$$
r^{2(2 l+2 h+2)}\|u\|_{l, 2 h+2, r}^{2} \leq C_{h, l}\left\{\|u\|_{0,0,2 r}^{2}+r^{2(2+2(l+3 h+1)+2 h)}\|f\|_{l+3 h+1,2 h, 2 r}^{2}\right\} .
$$

Proof. By means of left-translations and rescalings we can assume that $p_{o}=e$, and $r=1$. Using an induction argument and Theorem 3.5 one deduces that

$$
\left\|f_{i,(l, h)}^{\alpha}\right\|_{\gamma, v, 1} \leq C\|u\|_{l+1+\gamma, h-1+v, 2},
$$

and

$$
\left\|f_{(l, h)}^{\alpha}\right\|_{\gamma, v, 1} \leq C\left(\left\|f^{\alpha}\right\|_{l+\gamma, h+v, 2}+\|u\|_{l+1+\gamma, h+v, 2}+\|u\|_{l+2+\gamma, h-2+v, 2}\right) .
$$

Now choose a non-negative cut-off function $\eta_{1} \in C_{o}^{\infty}\left(B(e, 2), \mathbb{R}^{N}\right)$, with $\eta_{1}=1$ in $B(e, 1)$. Recall that $\eta \in C_{0}^{\infty}(B(0,2))$ is also non-negative. Using Theorem 3.2 we obtain

$$
\|\eta u\|_{l, h+2,1}^{2} \leq C\left(\|u\|_{h, l, 2}^{2}+\sum_{i=1}^{m}\left[\left\|f_{i,(l, h)}\right\|_{0,1,2}^{2}+\left\|f_{i,(l, h)}\right\|_{1,0,2}^{2}\right]+\left\|f_{(l, h)}\right\|_{1,0,2}^{2}\right) .
$$

From (3.11)-(3.12) one has

$$
\|u\|_{l, h+2,1}^{2} \leq C\left(\|u\|_{l+3, h+1,2}^{2}+\|f\|_{l+1, h, 2}^{2}\right) .
$$

Applying the energy inequality to (3.9) we obtain that for each $l$ and $h$ the following estimate holds

(3.15) $\left\|\eta_{1} v_{l, h+1}\right\|_{0,0,1}^{2} \leq C\left(\left\|v_{l, h}\right\|_{0,0,2}^{2}+i \sum_{i=1}^{m}\left\|f_{i,(l, h)}\right\|_{0,0,2}^{2}+\left\|f_{(l, h)}\right\|_{0,0,2}^{2}\right)$.

By (3.11)-(3.15) we infer

$$
\|\eta u\|_{l, h+2,1}^{2} \leq C\left(\|u\|_{l+3, h, 2}^{2}+\|f\|_{l+1, h, 2}^{2}\right) .
$$

If $h=2 \tilde{h}$, then iterating (3.16) $\tilde{h}$ times one finds

$$
\|u\|_{l, 2 \tilde{h}+2,1}^{2} \leq C_{h, l}\left(\|u\|_{l+3 \tilde{h}, 2,2}^{2}+\|f\|_{l+3 \tilde{h}-2,2 \tilde{h}, 2}^{2}\right) .
$$


On the other hand, if we take $l$-th order derivatives of the original system (3.7) in the directions $T_{1}, \ldots, T_{k} \in V^{2}$, we obtain the new system

$$
\sum_{\beta=1}^{N} \sum_{i, j=1}^{m} A_{i j}^{\alpha \beta} X_{i} X_{j} v_{l, 0}^{\beta}=f_{l, 0}^{\alpha}, \quad \alpha=1, \ldots, N
$$

Theorem 3.2 applied to (3.18) gives

$$
\|u\|_{l, 2,1}^{2} \leq C\left(\|u\|_{l, 1,2}+\|f\|_{l+1,0,2}\right) .
$$

Applying the energy inequality to (3.18) one may assume that in the right-hand side of the last inequality there is the norm $\|u\|_{l, 0,2}$, and not $\|u\|_{l, 1,2}$.

From a repeated application of Theorem 3.1 ( $l$ times) one concludes

$$
\|u\|_{l, 2,1}^{2} \leq C_{l}\left(\|u\|_{0,0,2}^{2}+\|f\|_{l+1,0,2}^{2}\right) .
$$

Now (3.10) follows at once from (3.17) and (3.19).

Remark. It is important to observe that as a consequence of (3.10) one obtains the hypoellipticity of the system

$$
\sum_{\beta=1}^{N} \sum_{i, j=1}^{m} A_{i j}^{\alpha \beta} X_{i} X_{j} u^{\beta}=0, \quad \alpha=1, \ldots, N .
$$

Hence, in the special setting of Carnot groups of step two, Theorem 3.6 generalizes to non-diagonal systems the basic results of Kohn $[\mathrm{K}]$ and Hörmander $[\mathrm{H}]$.

We now pass to the study of some consequences of Theorem 3.2 that will be useful in the study of the regularity of solutions of non-linear systems. The following corollaries are direct consequences of Theorem 3.2 and of the embedding of the Folland-Stein Sobolev spaces $\mathcal{L}^{h, 2}$ in the non-isotropic Hölder classes (see section two and thm 5.15 in $[\mathrm{F}]$ for details).

Corollary 3.7 Let $\mathbf{G}$ be a Carnot group of step two and $\Omega \subset \mathbf{G}$ be an open subset. If $u \in \mathcal{L}_{\text {loc }}^{1,2}(\Omega)$ is a solution of the constant-coefficient system

$$
\sum_{i, j=1}^{m} \sum_{\beta=1}^{N} A_{i j}^{\alpha \beta} X_{i} X_{j} u^{\beta}=0, \quad \alpha=1, \ldots, N
$$

in the gauge ball $B\left(p_{o}, 3 R\right) \subset \Omega$, then $u$ is smooth in $B\left(p_{o}, 3 R\right)$. Moreover, there exists a positive constant $C$ such that

$$
\sup _{B\left(p_{o}, R\right)}\left(|u|^{2}+R^{2}|X u|^{2}+R^{4} \sum_{i, j=1}^{m}\left|X_{i} X_{j} u\right|^{2}\right) \leq C \frac{1}{\left|B\left(p_{o}, 2 R\right)\right|} \int_{B\left(p_{o}, 2 R\right)}|u|^{2} d p
$$


Corollary 3.8 Let $\mathbf{G}, \Omega$ and $u$ be as in the previous corollary, then there exists a positive constant $C$ such that for each $0<r<R<2$ one has

$$
\int_{B\left(p_{o}, r\right)}\left|u-u_{o, r}\right|^{2} d p \leq C\left(\frac{r}{R}\right)^{Q+2} \int_{B\left(p_{o}, R\right)}\left|u-u_{o, R}\right|^{2} d p
$$

$$
\int_{B\left(p_{o}, r\right)}|u|^{2} d p \leq\left(\frac{r}{R}\right)^{Q} \int_{B\left(p_{o}, R\right)}|u|^{2} d p
$$

$$
\int_{B\left(p_{o}, r\right)}\left|X u-(X u)_{p_{o}, r}\right|^{2} d p \leq C\left(\frac{r}{R}\right)^{Q+2} \int_{B\left(p_{o}, R\right)}\left|X u-(X u)_{p_{o}, R}\right|^{2} d p
$$

$$
\int_{B\left(p_{o}, r\right)} \sum_{i, j=1}^{m}\left|X_{i} X_{j} u\right|^{2} d p \leq C\left(\frac{r}{R}\right)^{Q} \int_{B\left(p_{o}, R\right)} \sum_{i, j}^{m}\left|X_{i} X_{j} u\right|^{2} d p
$$

where $f_{p_{o}, r}$ denotes the average of the function $f$ on the ball $B\left(p_{o}, r\right)$.

Proof. The estimates (3.21), (3.22) follow from Corollary 3.7 similarly to the classical elliptic case. The key observation is that the function

$$
v(p)=u(p)-u_{p_{o}, R}-\sum_{i=1}^{m}\left(x_{i}(p)-x_{i}\left(p_{o}\right)\right)\left(X_{i} u\right)_{p_{o}, R}
$$

is still a solution of the system (3.20). This can be easily proved using the BakerCampbell-Hausdorff formula (2.1) (see also the explicit expression of $X_{i}^{\prime} s$ following (2.1)). Moreover, one has

$$
\int_{B\left(p_{o}, R\right)} v d p=0, \quad \int_{B\left(p_{o}, R\right)} X v d p=0 .
$$

The inequality (3.23) requires a new proof, since, because of the non-commutativity of the $X_{j}$ 's, we cannot simply say that $X_{i_{o}} u$ satisfies the same system as $u$. Instead, we have

$$
\sum_{\beta=1}^{N} \sum_{i, j=1}^{m} A_{i j}^{\alpha \beta} X_{i} X_{j}\left(X_{i_{o}} u^{\beta}\right)=\sum_{j=1}^{m} \sum_{\beta=1}^{N} c_{k, j}^{\alpha} X_{j} T_{k} u^{\beta}, \quad \alpha=1, \ldots, N .
$$

In order to deal with this system one has to make use of the results in Theorems 3.1 and 3.2, and Corollary 3.7. The first step consists in proving inequality (3.24). By homogeneity it suffices to do this for $R=1$, i.e.

$$
\int_{B\left(p_{0}, r\right)} \sum_{i, j=1}^{m}\left|X_{i} X_{j} u\right|^{2} d p \leq r^{Q} \int_{B\left(p_{0}, 1\right)} \sum_{i, j=1}^{m}\left|X_{i} X_{j} u\right|^{2} d p
$$


for $r<1$. In the case $0<r<1 / 2$, the latter inequality is obtained from Corollary 3.7 and the sub-elliptic Poincaré inequality, see $[\mathrm{J}]$ or [Va], as follows

$$
\begin{aligned}
& r^{-Q} \int_{B\left(p_{o}, r\right)} \sum_{i, j=1}^{m}\left|X_{i} X_{j} u\right|^{2} d p \\
& \quad=r^{-Q} \int_{B\left(p_{o}, r\right)} \sum_{i, j=1}^{m}\left|X_{i} X_{j} v\right|^{2} d p \leq C \sup _{B\left(p_{o}, 1 / 2\right)} \sum_{i, j=1}^{m}\left|X_{i} X_{j} v\right|^{2} \\
& \leq C \int_{B\left(p_{o}, 1\right)}|v|^{2} d p \leq C \int_{B\left(p_{o}, 1\right)}|X v|^{2} d p \leq C \int_{B\left(p_{o}, 1\right)} \sum_{i, j=1}^{m}\left|X_{i} X_{j} v\right|^{2} d p .
\end{aligned}
$$

The remaining case $1 / 2<r<1$ is trivial and requires no proof. This establishes (3.24). Once this is done formula (3.23) follows from the Caccioppoli inequality for $w=X_{i_{o}} u$ (see Theorem 3.2), and from the Poincaré inequality.

In the diagonal case proofs of (3.21)-(3.24) have appeared in [XZ].

Remark. In Sect. 5 we will also need the following estimate: Let $u \in \mathcal{L}_{\text {loc }}^{1,2}\left(\Omega, \mathbb{R}^{N}\right)$ be a solution of (3.20). There exists $C>0$ such that

$$
\int_{B(0, r)}|X u|^{2} d p \leq C\left(\frac{r}{R}\right)^{Q} \int_{B(0, R)}|X u|^{2} d p .
$$

This estimate too follows from Corollary 3.7 and from the Poincaré inequality.

To conclude the section we show how the method of difference quotients allows to establish $L^{2}$ estimates for some quasi-linear systems. Let $F: \mathbb{R}^{m N} \rightarrow \mathbb{R}^{+}$be a $C^{2}$ convex function satisfying the following structural assumption: There exists a positive constant $C$ such that for every $\xi \in \mathbb{R}^{m N}$ one has

$$
C^{-1}|\xi|^{2} \leq \sum_{i, j=1}^{m} \sum_{\alpha, \beta=1}^{N} \partial_{\xi_{i}^{\alpha}} \partial_{\xi_{j}^{\beta}} F(\xi) \leq C|\xi|^{2} .
$$

As an example, we can consider the functional in (1.2) when the gradient $|X u|$ is uniformly bounded by two positive constants.

Theorem 3.9 Let $u \in \mathcal{L}_{\mathrm{loc}}^{1,2}(\Omega)$, be a weak solution to the quasi-linear system

$$
\sum_{i=1}^{m} \int_{\Omega} F_{\xi_{i}^{\alpha}}(X u) X_{i} \phi^{\alpha} d p=0,
$$

for each $\phi \in C_{0}^{\infty}\left(\Omega, \mathbb{R}^{N}\right)$. The solution $u$ is weakly differentiable (in the $L^{2}$ sense) along the $V^{2}$ directions and moreover for each $i=1, \ldots, m$ and $j=1, \ldots, k$, we have

$$
X_{i} u, T_{j} u \in \mathcal{L}_{\mathrm{loc}}^{1,2}(\Omega) .
$$


Proof. The first step consists in differentiating (3.26) (in terms of difference quotients) along one of the $T_{k_{0}}$ directions, in the same spirit as (3.1). In this setting we will obtain the system

$$
\sum_{\beta=1}^{N} \sum_{i, j=1}^{m} \int_{\Omega} \partial_{\xi_{i}^{\alpha}} \partial_{\xi_{j}^{\beta}} F(X u)\left[X_{j} u^{\beta}\right]_{\left(T_{k_{0}}, \frac{1}{2}\right)} X_{i} \phi^{\alpha} d p=0
$$

for each $\phi \in \mathcal{L}^{1,2}\left(\Omega, \mathbb{R}^{N}\right)$. From the Caccioppoli inequality and Theorems 2.3 and 2.2, as in (3.3) we obtain the existence of fractional derivatives of order $\frac{1}{2}-\varepsilon$ in the $T_{k_{0}}$ direction

$$
\int_{\Omega}\left|\partial_{t_{k_{0}}}^{\frac{1}{2}-\varepsilon} X\left(\eta u^{\alpha}\right)\right|^{2} d p \leq C \int_{B(0,2)}\left(|u|^{2}+|X u|^{2}\right) d p .
$$

If we use Theorem 2.3 once more we obtain

$$
\int_{\Omega}\left|\partial_{t_{k_{0}}}^{1-2 \varepsilon}\left(\eta u_{h}^{\alpha}\right)\right|^{2} d p \leq C \int_{B(0,2)}\left(|u|^{2}+|X u|^{2}\right) d p .
$$

Repeating the argument in (3.5) we can easily prove that $T_{k_{0}} u$ exists in $L^{2}$ and moreover

$$
\int_{\Omega}\left|T_{k_{0}}\left(\eta u^{\alpha}\right)\right|^{2} d p \leq C \int_{B(0,2)}\left(|u|^{2}+|X u|^{2}\right) d p .
$$

To continue we differentiate the system (3.26) along $X_{j}$ with difference quotients of order one. As in Remark 3.4, commutators will appear. However, at this point we already have estimates on the commutators and this leads us directly to

$$
\int_{\Omega}\left|\left(X\left(\eta u^{\alpha}\right)\right)_{\left(X_{j}, 1\right)}\right|^{2} d p \leq C \int_{B(0,2)}\left(|u|^{2}+|X u|^{2}\right) d p .
$$

The proof is concluded once we apply Proposition 2.1. For more details we refer the reader to the paper [C1], where the scalar case was considered.

Since (3.26) is the Euler-Lagrange system of (1.1), the previous theorem has an immediate corollary.

Corollary 3.10 Let $F$ be as in Theorem 3.9. If $u \in \mathcal{L}_{\mathrm{loc}}^{1,2}(\Omega)$ is a critical point of the functional

$$
\mathcal{F}(u, \Omega)=\int_{\Omega} F(X u) d p,
$$

then $u$ is differentiable along the $V^{2}$ directions and moreover for each $i=1, \ldots, m$ and $j=1, \ldots, k$, we have

$$
X_{i} u, T_{j} u \in \mathcal{L}_{\mathrm{loc}}^{1,2}(\Omega)
$$




\section{Partial regularity of homogeneous systems and of local minimizers of convex functionals. The blow-up technique}

In this section using Theorem 3.6 we develop the blow-up analysis and prove partial regularity of weak solutions of non-linear systems of the form

$$
\sum_{\beta=1}^{N} \sum_{i, j=1}^{m} X_{i}\left(A_{i j}^{\alpha \beta}(x, u) X_{j} u^{\beta}\right)=0
$$

where $A_{i j}^{\alpha \beta}(x, u)$ are bounded continuous or uniformly continuous functions satisfying

$$
\sum_{\alpha, \beta=1}^{N} \sum_{i, j=1}^{m} A_{i j}^{\alpha \beta}(x, u) \xi_{i}^{\alpha} \xi_{j}^{\beta} \geq|\xi|^{2},
$$

for every $x \in \Omega$, and for every $N \times m$ matrix $\xi$. In section five we will allow in (4.1) a non zero right-hand side satisfying natural growth conditions. However, a method different from the one developed here will be needed, along with the additional hypothesis that $u$ be bounded. Before presenting the proof of the main theorem we need to introduce some preliminary definitions and results.

Definition. Let $\lambda>-\frac{Q-2}{2}$. A function $u \in L^{2}(\Omega)$ is said to belong to the Morrey space $\mathcal{M}^{2, \lambda}(\Omega)$ if

$$
r^{-Q} \int_{B(p, r) \cap \Omega}|u|^{2} d p \leq C r^{2(\lambda-1)},
$$

for every $p \in \Omega$ and $0<r<\operatorname{diam}(\Omega)$.

Definition. Let $\lambda \geq-\frac{Q}{2}$. A function $u \in L^{2}(\Omega)$ is said to belong to the Campanato space $\mathcal{C}^{2, \lambda}(\Omega)$ if for every $p \in \bar{\Omega}$ and $0<r<\operatorname{diam}(\Omega)$ one has $U\left(p_{0}, R\right) \leq C R^{2 \lambda}$, where we have let

$$
U\left(p_{0}, R\right)=\frac{1}{\left|B\left(p_{0}, R\right)\right|} \int_{B\left(p_{0}, R\right) \cap \Omega}\left|u(p)-u_{p_{0}, R}\right|^{2} d p .
$$

Remark. The classical characterization of Hölder continuous functions via integral means can be generalized to the setting of Carnot groups (see for instance [C2] or [Ga] and references therein). In particular, adapting the arguments in [DP] (see also Chapter 3, Theorem 1.2 in [Gi]), one has the following: Let $0<\lambda<1$. If $\Omega$ has the A-property, i.e., there exists $A>0$ such that for $R>0$ and $p \in \Omega$

$$
|\Omega \cap B(p, R)| \geq A|B(p, R)|,
$$

then $\mathcal{C}^{2, \lambda}(\Omega) \subset \Gamma_{\text {loc }}^{\lambda}(\Omega)$. In particular, the gauge pseudo-balls have the $A$-property (for a proof of this fact see for instance $[\mathrm{CG}]$ ), and therefore for every $p_{o} \in \mathbf{G}$, and every $r>0$ one has

$$
\mathcal{C}^{2, \lambda}\left(B\left(p_{o}, r\right)\right) \subset \Gamma_{\text {loc }}^{\lambda}\left(B\left(p_{o}, r\right)\right) \text { for } 0<\lambda<1 .
$$

This continuous inclusion will be henceforth tacitly used. 
Our first result in this section generalizes a beautiful theorem of Giusti and Miranda [GM].

Lemma 4.1 Let $u$ be a weak solution of (4.1), with continuous $A_{i j}^{\alpha \beta}$ 's. For each $0<\tau<1$, and $M>0$, there exist $\varepsilon_{o}$, and $R_{o}>0$ such that if for some $p_{o} \in \Omega$, one has $U\left(p_{o}, R\right)<\varepsilon_{o}^{2}$, and $\left|u_{p_{o}, R}\right| \leq M$ for $R \leq \min \left(R_{o}, d\left(p_{o}, \partial \Omega\right)\right)$, then

$$
U\left(p_{o}, \tau R\right) \leq C \tau^{2} U\left(p_{o}, R\right)
$$

Here, $C$ is the same as in (3.21).

Proof. We argue by contradiction, assuming that there exists $0<\tau<1, M>0$, sequences $\left(p_{n}\right) \in \Omega, \varepsilon_{n} \rightarrow 0, R_{n} \rightarrow 0$, and a sequence $u^{n}$ (the upper index is not an exponent) of weak solutions to (4.1), such that

$$
\begin{gathered}
\left|u_{p_{o}, R_{n}}^{n}\right| \leq M, \\
U^{n}\left(p_{n}, R_{n}\right)=\varepsilon_{n}^{2} \rightarrow 0,
\end{gathered}
$$

and

$$
U^{n}\left(p_{n}, \tau R_{n}\right)>2 C \tau^{2} \varepsilon_{n}^{2}
$$

Set

$$
v^{n}(q)=\varepsilon_{n}^{-1}\left[u^{n}\left(p_{n} \delta_{R_{n}}(q)\right)-u_{p_{n}, R_{n}}^{n}\right]
$$

From the homogeneity of the $X_{i}$ 's we have

$$
\left(X_{i} u^{n}\right)\left(p_{n} \delta_{R_{n}}(q)\right)=\epsilon_{n} R_{n}^{-1}\left(X_{i} v^{n}\right)(q) .
$$

For $R>0$, set $B_{R}=B(e, R)$, where $e$ is the group identity. From (4.1), and (4.6) we infer that for every $\phi \in C_{0}^{\infty}\left(B_{1}\right)$ the function $v^{n}$ satisfies the system

$$
\begin{array}{r}
\sum_{\beta=1}^{N} \sum_{i, j=1}^{m} \int_{B_{1}} A_{i j}^{\alpha \beta}\left(p_{n} \delta_{R_{n}}(q), \varepsilon_{n} v^{n}+u_{p_{n}, R_{n}}^{n}\right) X_{j}\left(v^{n}\right)^{\beta}(q) X_{i} \phi^{\alpha}(q) d q=0 \\
\alpha=1, \ldots, N
\end{array}
$$

Moreover, $v_{B_{1}}^{n}=0$,

$$
V^{n}(e, 1)=\frac{1}{\left|B_{1}\right|} \int_{B_{1}}\left|v^{n}\right|^{2} d q=1,
$$

and

$$
V^{n}(e, \tau)>2 C \tau^{2}
$$


From (4.8), taking a sub-sequence that we still denote as $v^{n}$, one obtains the existence of $v \in L^{2}\left(B_{1}\right)$ such that $v_{n} \rightarrow v$ weakly in $L^{2}\left(B_{1}\right)$, while $\varepsilon_{n} v^{n} \rightarrow 0$ a.e. in $B_{1}$. From the continuity assumptions on $A_{i j}^{\alpha \beta}$ and (4.2) one finds

$$
A_{i j}^{\alpha \beta}\left(p_{n} \delta_{R_{n}}(q), \varepsilon_{n} v^{n}+u_{p_{n}, R_{n}}^{n}\right) \rightarrow B_{i j}^{\alpha \beta}
$$

At this point we recall the compact embedding

$$
\mathcal{L}^{1,2}\left(B_{R}\right) \hookrightarrow \hookrightarrow L^{2}\left(B_{R}\right),
$$

noted in section two. This result, coupled with the Caccioppoli inequality Lemma 2.5, implies $X v^{n} \rightarrow X v$ weakly in $L^{2}\left(B_{1}\right)$ and $v^{n} \rightarrow v$ strongly in $L^{2}\left(B_{1}\right)$. All these facts, together with (4.9), give for every $\phi \in C_{0}^{\infty}\left(B_{1}\right)$

$$
\sum_{\beta=1}^{N} \sum_{i, j=1}^{m} \int_{B_{1}} B_{i j}^{\alpha \beta} X_{i}^{i} v^{\beta} X_{i}^{1} \phi^{\alpha} d p=0, \quad \alpha=1, \ldots, N .
$$

The contradiction now follows from (4.8), (4.9) and from (3.21).

Lemma 4.3 Choose $\tau^{2} \leq 2 C$ and let $u$ be a weak solution of (4.1) in $\Omega$. If at a point $p_{o} \in \Omega$ one has for some $R<R_{o}=R_{o}(M)$,

$$
\left|u_{p_{o}, R}\right|<M
$$

and

$$
U\left(p_{o}, R\right) \leq \eta_{o}^{2}(M)
$$

with $\eta_{o}(M) \leq \min \left\{\varepsilon_{o}(M), M \tau^{(Q+2) / 2}(1-\tau \sqrt{2 C})\right\}$, then for every integer $k$ we have

$$
U\left(p_{o}, \tau^{k} R\right) \leq\left(2 C \tau^{2}\right)^{k} U\left(p_{o}, R\right)
$$

We omit the proof of Lemma 4.3, since it is completely analogous to that of the elliptic case in [GM] (see also Chap. 4, p. 95, Theorem 1.1 in [Gi]). The key step is an iteration based on Lemma 4.1 .

Theorem 4.4 Let $u \in \mathcal{L}_{\mathrm{loc}}^{1,2}(\Omega)$ be a weak solution of (4.1) in $\Omega$. Denote by

$$
\begin{gathered}
S_{1}=\left\{p_{o} \in \Omega\left|\sup _{R}\right| u_{p_{o}, R} \mid=\infty\right\}, \\
S_{2}=\left\{p_{o} \in \Omega \mid \liminf _{R \rightarrow 0^{+}} U\left(p_{o}, R\right)>0\right\},
\end{gathered}
$$

and we let $S=S_{1} \cup S_{2}$ indicate the singular set. There exists $0<\alpha<1$ (depending only on $\mathbf{G}, \Omega$, and on the structural constants in (1.4)) such that $u \in \Gamma_{\text {loc }}^{0, \alpha}(\Omega \backslash S)$. 
Proof of Theorem 4.4. The proof of the Hölder regularity follows from Lemma 4.3, by adapting the arguments in [GM]. If $p \notin S$, then there exist $M>0$ and $\eta_{o}(M)$ such that in a small neighborhood $B\left(p, r_{o}\right)$ of $p$ the inequalities (4.11) and (4.12) hold. From Lemma 4.3 we conclude for each $R>0$ small enough and for some $0<a<1$

$$
U(p, R) \leq C R^{2 a}
$$

This implies $u \in \mathcal{C}^{2, a}\left(B\left(p, r_{o}\right)\right)$, the sub-elliptic Campanato class introduced above, and thereby the Hölder continuity of $u$ in $B\left(p, r_{o}\right)$ follows.

Our next goal is to estimate the Hausdorff dimension (with respect to the group pseudo-metric) of the singular set (compare with [Giu]).

Lemma 4.5 Let $u \in L_{\text {loc }}^{2}(\Omega)$. If (4.13) holds for some $a>0$ then $\lim _{R \rightarrow 0^{+}} u_{B(p, R)}$ exists and is finite. Consequently, $p$ is regular if and only if (4.13) holds for some $a>0$.

The elementary proof of Lemma 4.5 is left to the reader.

Remark. In the case where $A_{i j}^{\alpha \beta}$ are uniformly continuous the singular set coincides with $S_{2}$ and a point $p$ is regular if and only if (4.13) holds with $a=0$.

Lemma 4.6 Let $f \in L_{\mathrm{loc}}^{1}(\Omega)$ and set for $0<\alpha<Q$

$$
F_{\alpha}=\left\{p_{o} \in \Omega\left|\limsup _{r \rightarrow 0^{+}} r^{-\alpha} \int_{B\left(p_{o}, r\right)}\right| f(p) \mid d p>0\right\} .
$$

One has $H_{\alpha}\left(F_{\alpha}\right)=0$.

Proof. It suffices to show that, if for $s>0$ we let

$$
F_{S}=\left\{p_{o} \in \Omega\left|\limsup _{r \rightarrow 0^{+}} r^{-\alpha} \int_{B\left(p_{o}, r\right)}\right| f(p) \mid d p>s^{-1}\right\},
$$

then $H_{\alpha}\left(F^{s}\right)=0$. Let $K \subset \Omega$ be a compact set and consider an open set $O$ such that $K \subset O \subset \bar{O} \subset \Omega$. Fix $0<\varepsilon<D(K, \partial O)$. For every $p \in F_{s}$ there exists $r(p) \in(0, \varepsilon)$ such that

$$
r(p)^{-\alpha} \int_{B(p, r(p))}|f(q)| d q>s^{-1} .
$$

By the covering lemma of Wiener type in a space of homogeneous type (see [CW]) there exists a constant $\eta$, depending only on the homogeneous dimension $Q$, and a sequence of pairwise disjoint pseudo-balls $B_{i}=B\left(p_{i}, r\left(p_{i}\right)\right)$, such that $F^{s} \subset \bigcup_{i=1}^{\infty} \eta B_{i}$. One has

$$
\sum_{i=1}^{\infty} r_{i}^{\alpha} \leq s \int_{\bigcup_{i=1}^{\infty} B_{i}}|f(q)| d q
$$


and

$$
\left|\bigcup_{i=1}^{\infty} B_{i}\right| \leq C \eta \varepsilon^{Q-\alpha} s \int_{O}|f(q)| d q .
$$

Letting $\varepsilon \rightarrow 0$ in (4.15) we infer $H_{\alpha}\left(F^{s}\right)=0$. By the arbitrariness of $s>0$ the conclusion follows.

Theorem 4.7 Let $u$ be a weak solution of (4.1) in $\Omega$. For every $\varepsilon>0$ we have

$$
H_{Q-2+\varepsilon}(S)=0 \text {. }
$$

Moreover, if the coefficients $A_{i j}^{\alpha \beta}$ are uniformly continuous then one may take $\varepsilon=0$.

Proof. Set

$$
E_{\varepsilon}=\left\{p_{o} \in \Omega \mid \limsup _{r \rightarrow 0^{+}} r^{-\varepsilon} U\left(p_{o}, r\right)>0\right\} .
$$

Lemma 4.5 implies $S=\cap_{\varepsilon>0} E_{\varepsilon}$. We want to prove that for each $\varepsilon>0$

$$
H_{Q-2+\varepsilon}\left(E_{\varepsilon}\right)=0 .
$$

From the Poincaré inequality [J], [Va] one has

$$
r^{-\varepsilon} U\left(p_{o}, r\right) \leq C r^{-Q+2-\varepsilon} \int_{B\left(p_{o}, r\right)}|X u(p)|^{2} d p .
$$

Since $|X u|^{2}$ is in $L_{\text {loc }}^{1}(\Omega)$, the conclusion follows from Lemma 4.6.

Remark 4.8 It is easy to see that the solutions of (4.1) satisfy for some $r<2$ the following reverse Hölder inequality

$$
\int_{B_{1}}(1+|X u|)^{2} d p \leq\left(\int_{B_{2}}(1+|X u|)^{r} d p\right)^{\frac{2}{r}} .
$$

An adaptation of a well known argument due to Gehring (see [Ge], [BI]), based on the Calderón-Zygmund decomposition in spaces of homogeneous type (see [CW], [Ch]) allows to establish the higher integrability of $X u$ (see also [C3], [FSC], [GMo] and [GG3]). Since one can easily generalize Theorem 4.7 to the $L^{p}$ setting, with $p \geq 2$, then one infers that for some $p>2$

$$
H_{Q-p+\varepsilon}(S)=0 \text {. }
$$

We conclude this section with the proof of Theorem 1.1 which we will divide in two steps, Theorems 4.10 and 4.11. We stress that in the proof of Theorem 4.10 the convexity assumption on the energy function $F$, stated in the introduction, is only used in the study of the constant coefficient system arising as limit of the blow-ups of the Euler-Lagrange equations of (1.1). Such study was conducted in section three. Since the functional (1.1) is convex, then in particular it is strictly 
quasi-convex [E], i.e., there exists $\gamma>0$ such that for each $N \times m$ constant matrix $A$ and every $\phi \in C_{0}^{\infty}\left(\Omega, \mathbb{R}^{N}\right)$

$$
\int_{\Omega}\left[F(A)+\gamma\left(1+|X \phi|^{q-2}\right)|X \phi|^{2}\right] d p \leq \int_{\Omega} F(A+X \phi) d p,
$$

We can thus adapt to the present sub-Riemannian setting the ideas in L.C. Evans' paper [E] on quasi-convex elliptic integrands. In doing so, we will avoid any repetition, highlighting only those points where different arguments are needed. These arguments are collected in the following Propositions 4.8 and 4.9 which respectively contain a new Caccioppoli type inequality and the essence of the blow-up technique.

In the following, we will denote points $p \in \mathbf{G}$ by exponential coordinates $p=(x, t)$ where $x \in V^{1}$ and $t \in V^{2}$. Observe that if $A$ is an $N \times m$ matrix, then $X(A \cdot x)(p)=A$ for every $p \in \mathbf{G}$. If $f$ is a function, we let $f_{\Omega} f(p) d p=$ $\frac{1}{|\Omega|} \int_{\Omega} f(p) d p$.

Proposition 4.9 Let $u \in \mathcal{L}_{\text {loc }}^{1, q}(\Omega)$ be a local minimizer of (1.1). For each $L>0$ there exists $C_{1}(L)>0$ such that

$$
f_{B\left(p_{o}, r\right)}\left(1+|X u-A|^{q-2}\right)|X u|^{2} d p \leq
$$

$C(L)\left(\frac{1}{r^{2}} \int_{B\left(p_{o}, r\right)}\left|u(p)-a-A \cdot\left(x-x_{o}\right)\right|^{2} d p\right.$

$$
\left.+\frac{1}{r^{q}} \int_{B\left(p_{o}, r\right)}\left|u(p)-a-A \cdot\left(x-x_{o}\right)\right|^{q} d p\right),
$$

for each $B\left(p_{o}, 2 r\right) \subset \Omega, a \in \mathbb{R}^{N}$, and any $N \times m$ constant matrix $A$ with $|A| \leq L$.

Proof. Without loss of generality we can assume $p_{o}=e$, the group identity. For $r \leq t<s \leq 2 r$ choose a test function $\xi \in C_{o}^{\infty}\left(B\left(p_{o}, s\right)\right)$, with $\xi=1$ in $B\left(p_{o}, t\right)$. Define two auxiliary functions $\phi(p)=\xi(u-a-A \cdot x)$, and $\varphi(p)=$ $(1-\xi)(u-a-A \cdot x)$. Following Evans [E], and using (4.17) we can easily prove

$$
\begin{gathered}
\int_{B(e, s)}\left[F(A)+\gamma\left(1+|X \phi|^{q-2}\right)|X \phi|^{2}\right] d p \leq \\
\int_{B(e, s)}\left[F(X u)-F^{\prime}(X u) X \varphi+C\left(1+|X u|^{q-2}+|X \varphi|^{q-2}\right)|X \varphi|^{2}\right] d p .
\end{gathered}
$$

Since $u$ is a minimizer of (1.1), using $|A| \leq L$, and $D^{2} F(X u) \leq C(1+|X u|)^{q-2}$, we obtain

$$
\int_{B(e, s)} F(X u) d p \leq \int_{B(e, s)}\left[F(A)+F^{\prime}(A) X \varphi+C\left((1+|X u|)^{q-2}|X \varphi|^{2}\right)\right] d p .
$$

The latter inequality and (4.19) give 


$$
\gamma \int_{B(e, s)}\left(1+|X \phi|^{q-2}\right)|X \phi|^{2} d p
$$

$$
\leq C \int_{B(e, s)}\left[(1+|X u|)^{q-2}|X u-A \| X \varphi|\right.
$$

$$
\left.+C\left(1+|X u|^{q-2}+|X \varphi|^{q-2}\right)|X \varphi|^{2}\right] d p .
$$

Recalling the definition of $\xi$ and using (4.20) we easily arrive at

$$
\begin{gathered}
\int_{B(e, t)}\left(1+|X u-A|^{q-2}\right)|X u|^{2} d p \\
\leq C\left\{\int_{B(e, s) \backslash B(0, t)}(1+|X u-A|)^{q-2}|X u|^{2} d p\right. \\
\left.+\int_{B(e, 2 r)}\left[\frac{|u-a-A \cdot x|^{2}}{(s-t)^{2}}+\frac{|u-a-A \cdot x|^{q}}{(s-t)^{q}}\right] d p\right\} .
\end{gathered}
$$

The conclusion follows from a standard argument based on the so-called hole filling technique (se [Gi] or [E, Lemma 5.1 on p. 239]).

Proposition 4.10 Let $u$ be as in Proposition 4.8. For each $L>0$, there exists $C_{2}(L)>0$ such that for every $0<\theta<1 / 4$ one can find $\varepsilon(\theta, L)>0$ for which, if $\left|(X u)_{p_{o}, r}\right| \leq L,\left|(X u)_{p_{o}, \theta r}\right| \leq L$, and $U\left(p_{o}, r\right) \leq \varepsilon$, then

$$
U\left(p_{o}, \theta r\right) \leq C_{2}(L) \theta^{2} U\left(p_{o}, r\right),
$$

where

$$
U\left(p_{o}, r\right)=f_{B\left(p_{o}, r\right)}\left(1+\left|X u-(X u)_{p_{o}, r}\right|\right)^{q-2}\left|X u-(X u)_{p_{o}, r}\right|^{2} d p .
$$

Proof. Similarly to Lemma 4.3 the proof proceeds by contradiction. If the assertion of the proposition were false, then there would exist a sequence of balls $B\left(p_{k}, r_{k}\right) \subset \Omega$, such that $\left|(X u)_{p_{k}, r_{k}}\right| \leq L,\left|(X u)_{p_{k}, \theta r_{k}}\right| \leq L, U\left(p_{k}, r_{k}\right)=\lambda_{k}^{2} \rightarrow 0$ as $k \rightarrow \infty$, and

$$
U\left(p_{k}, \theta r_{k}\right)>C_{2}(L) \theta^{2} \lambda_{k}^{2} .
$$

We now consider the blow-up sequence defined by

$$
v^{k}(p)=\frac{u\left(p_{k} \delta_{r_{k}}(p)\right)-(u)_{p_{k}, r_{k}}-(X u)_{p_{k}, r_{k}} \cdot x}{\lambda_{k} r_{k}} .
$$

By a simple rescaling, using the fact that the vector fields $X_{i}$ 's are homogeneous of degree one with respect to the non-isotropic group dilations, one obtains from (4.21) the inequality

$$
f_{B(e, \theta)}\left(1+\left|\lambda_{k}^{q-2}\right| X v^{k}-\left.\left(X v^{k}\right)_{e, \theta}\right|^{q-2}\right)\left|X v^{k}-\left(X v^{k}\right)_{e, \theta}\right|^{2} d p>C_{2}(L) \theta^{2} .
$$


Using the compact embedding $\mathcal{L}^{1,2}(B(e, 2)) \hookrightarrow \hookrightarrow L^{2}(B(e, 2))$, one obtains the existence of $v \in \mathcal{L}^{1,2}(B(e, 2))$, such that $v^{k} \rightarrow v$ weakly in $\mathcal{L}^{1,2}(B(e, 2))$, and strongly in $L^{2}(B(e, 2))$. Moreover, $(X u)_{p_{k}, r_{k}} \rightarrow A \in \mathbb{R}^{N \times m}$. Repeating the argument in [E, Lemma 6.2], we infer that $v$ is a weak solution of a constant coefficient sub-elliptic system of the form (4.10), with coefficients depending on $A$. This system satisfies (1.7) since the original functional is convex. It is at this point that the sub-elliptic estimates from section three enter into play. In particular, by means of Corollary (3.7) and the Poincaré inequality [J], [Va], one has

$$
f_{B(e, 2 \theta)}\left|X v-(X v)_{B(e, \theta)}\right|^{2} \leq C(L) \theta^{2}
$$

(note that the average in the integral is on a smaller ball). Using the latter inequality and the Sobolev-Poincaré inequalities recalled in section two, one obtains

$$
\lim _{k \rightarrow \infty} \lambda_{k}^{q-2} f_{B(e, 2 \theta)}\left|v^{k}-\left(v^{k}\right)_{e, 2 \theta}-\left(X v^{k}\right)_{e, \theta} \cdot x\right|^{q} d p=0 .
$$

The inequality (4.18) in Proposition 4.8, and (4.23), finally give

$\limsup _{k \rightarrow \infty} f_{B(e, \theta)}\left(1+\left|\lambda_{k}^{q-2}\right| X v^{k}-\left.\left(X v^{k}\right)_{e, \theta}\right|^{q-2}\right)\left|X v^{k}-\left(X v^{k}\right)_{e, \theta}\right|^{2} d p \leq C_{3}(L) \theta^{2}$.

Since the latter inequality contradicts (4.22), we have completed the proof.

Theorem 4.11 Let $u \in \mathcal{L}_{\mathrm{loc}}^{1, q}(\Omega)$ be a local minimizer of (1.1). There exists a closed subset $S \subset \Omega$, with $|S|=0$, such that Xu is locally Hölder continuous in $\Omega \backslash S$.

Proof. With Propositions 4.8 and 4.9 in hands, the proof of Theorem 4.10 follows along the lines of that of Lemma 4.3 without major modifications. For more details we refer the reader to Evans' paper [E].

Proposition 4.9 characterizes the singular set $S$. One has $S=S_{1} \cup S_{2}$, where

$$
S_{1}=\left\{p_{o} \in \Omega\left|\lim _{R \rightarrow 0^{+}} R^{-Q} \int_{B\left(p_{o}, R\right)}\right| X u-\left.X u_{p_{o}, R}\right|^{q} d p>0\right\},
$$

and

$$
S_{2}=\left\{p_{o} \in \Omega \mid \lim _{R \rightarrow 0^{+}} X u_{p_{o}, R} \text { does not exist }\right\} .
$$

Clearly, $S$ has measure zero. In the case $q=2$ we can say more about the singular set.

Theorem 4.12 Let $u$ be as in Theorem 4.10, with $q=2$. For every $\varepsilon>0$ one has

$$
H_{Q-2+\varepsilon}(S)=0 .
$$


Proof. By virtue of Theorem 3.9 and Corollary 3.10 we have that for each $i=$ $1, \ldots, m$ and $j=1, \ldots, k$

$$
X_{i} u, T_{j} u \in \mathcal{L}_{\mathrm{loc}}^{1,2}(\Omega) .
$$

The conclusion now follows from an argument similar to that in Theorem 4.7. One needs to use the results from Lemma 4.5, 4.6, the characterization of the singular set from Proposition 4.10, the Poincaré inequality (see [J], [Va]), and (4.26).

Theorem 4.11 generalizes a result of Giusti and Miranda [GM].

\section{Partial regularity for non-homogeneous systems via the Morrey-Campanato approach}

In this section we establish the partial regularity of weak solutions of (1.3). Our results generalize to the sub-Riemannian setting those of Giaquinta and Giusti in [GG2]. Here, in order to treat systems whose right hand side satisfies the natural growth conditions (1.5), we need to assume a priori the boundedness of the weak solution $|u| \leq M$. In connection with (1.4), (1.5), we also assume

$$
a(M) M \leq \lambda(M) / 2 .
$$

For the necessity of (5.1) see [Gi] Remark 1.3, Chap. 3. Since for the most part the proofs of the results in this section follow closely those of their Euclidean predecessors, we provide only sketches of the arguments, emphasizing only the parts where the results of section three are used.

We begin by introducing several preliminary results. The first is a maximum principle for constant coefficients systems which extends a result of Cannarsa [Ca].

Lemma 5.1 Let $\phi \in \mathcal{L}_{\text {loc }}^{1,2}(B(e, 1)) \cap L^{\infty}(B(e, 1))$, and assume that $X \phi \in$ $\mathcal{M}^{2,0}(B(e, 1))$. Set

$$
L^{2}=\sup _{p_{0} \in \overline{B(e, 1)}, r>0} r^{2-Q} \int_{B\left(p_{0}, r\right) \cap B(e, 1)}|X \phi|^{2} d p<\infty .
$$

Let $v \in \mathcal{L}_{\mathrm{loc}}^{1,2}(B(e, 1))$ be a weak solution in $B(e, 1)$ of the constant coefficient system

$$
\sum_{i, j=1}^{m} \sum_{\beta=1}^{N} A_{i j}^{\alpha \beta} X_{i} X_{j} v^{\beta}=0
$$

with $v-\phi \in \mathcal{L}_{0}^{1,2}(B(e, 1))$, and $A_{i j}^{\alpha \beta}$ satisfying the coercivity condition (1.7). There exists a constant $C>0$ independent of $v$, and $\phi$ such that

$$
\sup _{B(e, 1)}|v| \leq C\left(\sup _{B(e, 1)}|\phi|+L\right) .
$$


Proof. The proof is divided in two parts. First we show that

(i) $\sup _{B(e, 1)}|v|^{2} \leq C\left(\sup _{B(e, 1)}|\phi|^{2}+L^{2}+\sup _{p_{o} \in \overline{B(e, 1)}, r>0} r^{2-Q} \int_{B\left(p_{0}, r\right) \cap B(e, 1)}|X v|^{2} d p\right)$.

To conclude the proof we then establish the following Campanato-type estimate

$$
\sup _{p_{o} \in \overline{B(e, 1)}, r>0} r^{2-Q} \int_{B\left(p_{o}, r\right) \cap B(e, 1)}|X v|^{2} d p \leq C L^{2} .
$$

In order to prove (i) let $p_{o} \in B(e, 1)$, and set $d=d\left(p_{o}, \partial B(e, 1)\right)=d\left(p_{o}, z_{o}\right)$ for some $z_{o} \in \partial B(e, 1)$. In virtue of (3.22) for any $0<r<R=\min (d, 1)$ one has

$$
\begin{gathered}
r^{-Q} \int_{B\left(p_{o}, r\right)}|v|^{2} d p \leq C R^{-Q} \int_{B\left(p_{o}, R\right)}|v|^{2} d p \leq \\
C\left[\sup _{B(e, 1)}|\phi|^{2}+R^{-Q} \int_{B\left(z_{o}, 2 R\right) \cap B(e, 1)}|\phi-v|^{2} d p\right] .
\end{gathered}
$$

From the Poincaré inequality we infer

$$
r^{-Q} \int_{B\left(p_{o} \omega, r\right)}|v|^{2} d p \leq C\left[\sup _{B(e, 1)}|\phi|^{2}+L^{2}+R^{2-Q} \int_{B\left(z_{o}, 2 R\right) \cap B(e, 1)}|X v|^{2} d p\right] .
$$

The latter inequality gives the estimate (i). In order to prove (ii) we observe that the function $w=\phi-v \in \mathcal{L}_{o}^{1,2}(B(e, 1))$ is a weak solution of the system

$$
\sum_{i, j=1}^{m} \sum_{\beta=1}^{N} A_{i j}^{\alpha \beta} X_{i} X_{j} w=\sum_{i=1}^{m} X_{i} F_{i}^{\alpha} \alpha=1, \ldots, N
$$

where

$$
F_{i}^{\alpha}:=\sum_{j=1}^{m} \sum_{\beta=1}^{N} A_{i j}^{\alpha \beta} X_{j} \phi \alpha=1, \ldots, N
$$

in $B(e, 1)$. Let $p_{o} \in B(e, 1)$, and $r>0$. Choose a test function $\eta \in C_{o}^{\infty}\left(B\left(p_{o}, 2 r\right)\right)$, such that $\eta=1$ in $B\left(p_{o}, r\right)$ and $|X \eta| \leq C r^{-1}$. If we multiply both sides of the latter equation by $w \eta^{2}$ and integrate by parts, then by (1.7) we obtain the following energy inequality

$$
\begin{aligned}
\int_{B\left(p_{o}, r\right) \cap B(e, 1)}|X \phi-X v|^{2} d p & \leq C \int_{B\left(p_{o}, 2 r\right) \cap B(e, 1)}|F|^{2} d p \\
& \leq C \int_{B\left(p_{o}, 2 r\right) \cap B(e, 1)}|X \phi|^{2} d p .
\end{aligned}
$$

From Schwarz inequality one infers

$$
\int_{B\left(p_{o}, r\right) \cap B(e, 1)}|X v|^{2} d p \leq C \int_{B\left(p_{o}, 2 r\right) \cap B(e, 1)}|X \phi|^{2} d p .
$$

The estimate (ii) follows from the latter inequality, once we multiply both sides by $r^{2-Q}$ and take the supremum over all gauge balls $B\left(p_{o}, r\right)$. 
One of the main ingredients in the proof of partial regularity for non-homogeneous systems is the higher integrability of the gradient of the solution.

Lemma 5.2 Let $u \in \mathcal{L}_{\text {loc }}^{1,2}(\Omega)$ be a bounded weak solution of (1.3), and assume that (1.4), (1.5) and (5.1) hold. There exists $q>2$ such that for every $B\left(p_{o}, 2 r\right) \subset \Omega$ one has

$$
\|X u\|_{L^{q}\left(B\left(p_{o}, r\right)\right)} \leq r^{Q\left(\frac{1}{q}-\frac{1}{2}\right)} C\|(1+|X u|)\|_{L^{2}\left(B\left(p_{o}, 2 r\right)\right)} .
$$

Moreover, if the right hand side of (1.3) is zero (i.e. $a(M)=b=0$ ), then one has

$$
\|X u\|_{L^{q}\left(B\left(p_{o}, r\right)\right)} \leq r^{Q\left(\frac{1}{q}-\frac{1}{2}\right)} C\|X u\|_{L^{2}\left(B\left(p_{o}, 2 r\right)\right)} .
$$

Proof. The hypothesis (1.4), (1.5) allow to prove a reverse Hölder inequality for the function $(1+|X u|)$. The proof is standard and rests on the Caccioppoli and Poincaré-Sobolev inequalities in section two. Once we have the reverse Hölder inequality, then a well-known argument (see [Ge], [BI], [FSC] and Chap. 5 in [Gi]), yields the higher integrability of the horizontal gradient. In the homogeneous case, when $a(M)=b=0$, the reverse Hölder inequality can be proved directly for the function $|X u|$, without need of the additional constant. The higher integrability argument mentioned above yields inequality (5.3) (see also Remark 4.8 for more references).

The next lemma is the main step in the regularity proof, it substitutes the blow-up argument in section four. We shall suppose that the coefficients $A_{i j}^{\alpha \beta}$ are continuous functions of their arguments, and we shall denote by $\omega(r)$ a bounded continuous function such that

$$
|A(x, u)-A(y, v)| \leq \omega\left(|x-y|^{2}+|u-v|^{2}\right),
$$

whenever $x, y \in \Omega$ and $|u|,|v| \leq M$. Without loss of generality we may assume that $\omega$ is increasing, concave and $\omega(0)=0$ (unless the coefficients $A$ are uniformly continuous one cannot expect $\omega$ to be continuous).

Lemma 5.3 Let $\mathbf{G}$ be a Carnot group of step two and $\Omega \subset \mathbf{G}$ an open set. Let $u \in \mathcal{L}_{\mathrm{loc}}^{1,2}(\Omega)$ be a bounded weak solution of (1.3), and assume that (1.4), (1.5) and (5.1) hold. There exists $R_{0}=R_{0}(M, a, b)>0$ such that for each point $p_{0} \in \Omega$ and for every $0<r<R<\min \left(R_{0}, d\left(p_{0}, \partial \Omega\right)\right)$, one has

$$
\int_{B\left(p_{0}, r\right)}\left(1+|X u|^{2}\right) d p \leq C \int_{B\left(p_{0}, R\right)}\left(1+|X u|^{2}\right) d p\left[\left(\frac{r}{R}\right)^{Q}+\chi\left(p_{0}, R\right)\right]
$$

where

$$
\chi\left(p_{0}, R\right)=R+\phi(R)^{\frac{q-2}{2 q}}+\omega(C \phi(R))^{\frac{q-2}{q}},
$$

and $\phi(R)=R^{2-Q} \int_{B\left(p_{0}, R\right)}\left(1+|X u|^{2}\right) d p$, with $q>2$ is as in (5.2). 
Proof. First we prove that there exists $R>0$ such that

$$
\sup _{r<R, B\left(p_{0}, r\right) \subset \Omega} r^{2-Q} \int_{B\left(p_{0}, r\right)}|X u|^{2} d p \leq C \sup _{\Omega}|u| .
$$

Let $B\left(p_{0}, 2 r\right) \subset \Omega$. Set $\phi=\eta^{2} u$, with $\eta \in C_{0}^{\infty}\left(B\left(p_{0}, 2 r\right)\right) \eta=1$ in $B\left(p_{0}, r\right)$, $|X \eta| \leq \mathrm{Cr}^{-1}$ and use it as a test function in the weak form of (1.3),

$$
\begin{aligned}
& \sum_{\beta=1}^{N} \sum_{i, j=1}^{m} \int_{B\left(p_{0}, 2 r\right)}\left(A_{i j}^{\alpha \beta}(x, u) X_{j} u^{\beta}\right) X_{i} \phi^{\alpha}(x) d x \\
& \quad=\int_{B\left(p_{0}, 2 r\right)} f^{\alpha}(x, u, X u) \phi^{\alpha}(x) d x . \alpha=1, \ldots, N
\end{aligned}
$$

By means of (1.4), and (1.5) we obtain the Caccioppoli inequality

$$
\begin{gathered}
\int_{B\left(p_{0}, 2 r\right)}|X u|^{2} \eta^{2}(x) d x \leq C r^{2} \int_{B\left(p_{0}, 2 r\right)}|u|^{2}(x) d x+\int_{B\left(p_{0}, 2 r\right)}|f|(x)|u|(x) \eta^{2}(x) d x . \\
\leq C r^{2} \int_{B\left(p_{0}, 2 r\right)}|u|^{2}(x) d x+\int_{B\left(p_{0}, 2 r\right)}\left(a(M)|u||X u|^{2} \eta^{2}+b(M)|u| \eta^{2}\right) d x .
\end{gathered}
$$

Now we use (5.1) and obtain for every $p \in \Omega$ and small $r>0$,

$$
\int_{B(p, r)}|X u|^{2} \leq C r^{Q-2}\left[M^{2}+b r^{2} M\right] .
$$

The estimate (5.5) follows from (5.6) if we choose $R>0$ small enough so that $b r^{2} \leq M$. Inequality (5.4) is obvious for $\frac{R}{2}<r<R$, hence we will consider only the case $0<r<\frac{R}{2}$. of

Next, we split $u$ as $u=v+w$, where $v \in \mathcal{L}_{\text {loc }}^{1,2}\left(B\left(p_{0}, R / 2\right), \mathbb{R}^{n}\right)$ is a solution

$$
\sum_{i, j=1}^{2 n} \sum_{\beta=1}^{N} A_{i j}^{\alpha \beta}\left(p_{0},(u)_{p_{0}, R / 2}\right) X_{i} X_{j} v^{\beta}=0,
$$

with boundary data $v-u \in \mathcal{L}_{0}^{1,2}\left(B\left(p_{0}, R / 2\right)\right)$.

Recall that by (3.25) we have that for any $0<r<R / 2$ the following inequality holds

$$
\int_{B(0, r)}|X v|^{2} d x \leq C\left(\frac{r}{R}\right)^{Q} \int_{B\left(p_{0}, R / 2\right)}|X v|^{2} d x .
$$

From Lemma 5.1 and (5.5) one obtains

$$
\sup _{B\left(p_{0}, R / 2\right)}|v| \leq C M .
$$

From (5.7) we deduce that $|w| \leq C M$. 
Observe that $w \in \mathcal{L}_{0}^{1,2}\left(B\left(p_{0}, R / 2\right), \mathbb{R}^{n}\right)$, is a weak solution of the system

$$
\begin{gathered}
\sum_{\beta=1}^{N} \sum_{i, j=1}^{m} \int_{B\left(p_{0}, R / 2\right)}\left(A_{i j}^{\alpha \beta}\left(p_{0}, u_{p_{0}, R / 2}\right) X_{j} w^{\beta}\right) X_{i} \phi^{\alpha}(x) d x \\
=\int_{B\left(p_{0}, R / 2\right)}\left(A_{i j}^{\alpha \beta}\left(p_{0}, u_{p_{0}, R / 2}\right)-A_{i j}^{\alpha \beta}(x, u)\right) X_{j} w^{\beta} X_{i} \phi^{\alpha}(x) d x \\
+\int_{B\left(p_{0}, R / 2\right)} f^{\alpha}(x, u, X u) \phi^{\alpha}(x) d x . \alpha=1, \ldots, N
\end{gathered}
$$

Set $\phi=w$ and we obtain the Caccioppoli inequality

$$
\int_{B\left(p_{0}, R / 2\right)}|X w|^{2} d p \leq C\left[\int_{B\left(p_{0}, R / 2\right)}\left(\omega^{2}+|w|\right)|X u|^{2}+\int_{B\left(p_{0}, R / 2\right)}|w| d p\right],
$$

where $\omega^{2}=\omega^{2}\left(R^{2}+\left|u-u_{p_{0}, R / 2}\right|^{2}\right)$.

From the Poincaré inequality we have

$$
\begin{gathered}
\frac{1}{\left|B\left(p_{0}, R / 2\right)\right|} \int_{B\left(p_{0}, R / 2\right)}|w| d x \leq C R \frac{1}{\left|B\left(p_{0}, R / 2\right)\right|} \int_{B\left(p_{0}, R / 2\right)}|X w| d x \\
\leq C R\left(\varepsilon \int_{B\left(p_{0}, R / 2\right)}|X w|^{2} d x+\frac{1}{\varepsilon}\right) .
\end{gathered}
$$

From (5.8) and the Poincaré inequality we obtain

$$
\frac{1}{\left|B\left(p_{0}, R / 2\right)\right|} \int_{B\left(p_{0}, R / 2\right)}|w| d x \leq C R\left(\frac{1}{\left|B\left(p_{0}, R / 2\right)\right|} \int_{B\left(p_{0}, R / 2\right)}\left(1+|X u|^{2}\right) d x\right) .
$$

To complete the estimate of the terms in (5.8) we use the results in Lemma 5.2 and obtain

$$
\frac{1}{\left|B\left(p_{0}, R / 2\right)\right|} \int_{B\left(p_{0}, R / 2\right)} \omega^{2}|X u|^{2} d x
$$

$$
\leq C \frac{1}{\left|B\left(p_{0}, R\right)\right|} \int_{B\left(p_{0}, R\right)}\left(1+|X u|^{2}\right) d x\left(\frac{1}{\left|B\left(p_{0}, R / 2\right)\right|} \int_{B\left(p_{0}, R / 2\right)} \omega^{2 q /(q-2)} d x\right)^{\frac{q-2}{q}}
$$

where $q>2$ is as in (5.2). Since $\omega$ is bounded we have $\omega^{2 q /(q-2)} \leq C \omega$. Being $\omega$ concave, then Jensen inequality and (5.10) yield 


$$
\frac{1}{\left|B\left(p_{0}, R / 2\right)\right|} \int_{B\left(p_{0}, R / 2\right)} \omega^{2}|X u|^{2} d x
$$

$$
\begin{aligned}
\leq C \frac{1}{\left|B\left(p_{0}, R\right)\right|} \int_{B\left(p_{0}, R\right)}\left(1+|X u|^{2}\right) d x & \\
& \times \omega\left(C R^{2}+\frac{1}{\left|B\left(p_{0}, R\right)\right|} \int_{B\left(p_{0}, R\right)}\left|u-u_{p_{0}, R}\right|^{2} d x\right)
\end{aligned}
$$

$\leq C \frac{1}{\left|B\left(p_{0}, R\right)\right|} \int_{B\left(p_{0}, R\right)}\left(1+|X u|^{2}\right) d x$

$$
\times \omega\left(C R^{2} \frac{1}{\left|B\left(p_{0}, R\right)\right|} \int_{B\left(p_{0}, R\right)}\left(1+|X u|^{2}\right) d x\right) .
$$

Similarly we obtain

$$
\frac{1}{\left|B\left(p_{0}, R / 2\right)\right|} \int_{B\left(p_{0}, R\right)} w|X u|^{2} d x
$$

$$
\leq C \frac{1}{\left|B\left(p_{0}, R\right)\right|} \int_{B\left(p_{0}, R\right)}\left(1+|X u|^{2}\right) d x\left(\frac{1}{\left|B\left(p_{0}, R\right)\right|} \int_{B\left(p_{0}, R\right)}|w|^{2} d x\right)^{\frac{q-2}{q}},
$$

where the constant $C$ depends on $M$ as well. At this point we recall (5.8), that together with (5.9)-(5.12) and the Poincaré inequality yields

$$
\int_{B\left(p_{0}, R / 2\right)}|X w|^{2} d p \leq C \chi\left(p_{0}, R\right) \int_{B\left(p_{0}, R\right)}\left(1+|X u|^{2}\right) d p .
$$

To conclude the proof we use (3.25) and obtain for $0<r<\frac{R}{2}$,

$$
\begin{gathered}
\int_{B\left(p_{0}, r\right)}\left(1+|X u|^{2}\right) d x \leq C\left[\int_{B\left(p_{0}, r\right)}\left(1+|X v|^{2}\right) d x+\int_{B\left(p_{0}, r\right)}|X w|^{2} d x\right] \\
\leq C\left[\left(\frac{r}{R}\right)^{Q} \int_{B\left(p_{0}, R / 2\right)}\left(1+|X v|^{2}\right) d x+\int_{B\left(p_{0}, R / 2\right)}|X w|^{2} d x\right] .
\end{gathered}
$$

The conclusion follows at once from the latter, from the argument in the proof of (ii) in Lemma 5.1, and from (5.13).

We are now ready to prove the main result of the section.

Theorem 5.4 Let $\mathbf{G}$ be a Carnot group of step two and $\Omega \subset \mathbf{G}$ an open set. Let $u \in \mathcal{L}_{\text {loc }}^{1,2}(\Omega)$ be a bounded weak solution of (1.3), and assume that (1.4), (1.5) and (5.1) hold. There exists a closed set $S \subset \Omega$ such that $u$ is $\alpha-H o ̈ l d e r$ continuous in $\Omega \backslash S$ for every $0<\alpha<1$ and $H_{Q-2-\varepsilon}(S)=0$ for some $\varepsilon \geq 0$. 
Proof. From Lemma 5.3 one has that for $0<\theta<1$ the following estimate holds

$$
\phi(\theta R) \leq C \theta^{2} \phi(R)\left(1+\chi\left(p_{0}, R\right) \theta^{-Q}\right) .
$$

Fix $\alpha \in(0,1)$. Choose $\theta$ in such a way that $2 C \theta^{2-2 \alpha}-1$. If, for some $R>0$ small enough

$$
\chi\left(p_{0}, R\right)<\theta^{Q}
$$

then we can iterate (5.14) starting from

$$
\phi(\theta R) \leq C \tau^{2 \alpha} \phi(R)
$$

and obtain

$$
\phi\left(\theta^{k} R\right) \leq C \tau^{2 k \alpha} \phi(R)
$$

which implies

$$
\int_{B\left(p_{0}, r\right)}|X u|^{2} d x \leq C r^{Q-2+2 \alpha}\left[\int_{\Omega}\left(1+|X u|^{2}\right) d x\right] .
$$

From the latter and the Poincaré inequality it follows that $u$ is $\alpha$-Hölder continuous in a neighborhood of $p_{0}$. The singular set of $u$ is characterized by inequality (5.15), from which it is easy to infer that

$$
S=\left\{\left.p_{0} \in \Omega\left|\liminf _{R \rightarrow 0^{+}} R^{2-Q} \int_{B\left(p_{0}, R\right)}\right| X u\right|^{2} d p>0\right\} .
$$

The estimate on the Hausdorff dimension of $S$ can be proved arguing as in Theorem 4.7.

Theorem 5.1 holds for coefficients which are merely continuous. When $A_{i j}^{\alpha \beta}$ are Hölder continuous, then the horizontal derivatives of $u$ are Hölder continuous too.

Theorem 5.5 Let $u, \Omega$ and $S$ be as in Theorem 5.4. If $A_{i j}^{\alpha \beta}$ are Hölder continuous with exponent $\sigma$, then $X u$ is locally Hölder continuous with the same Hölder exponent in the set $\Omega \backslash S$.

Proof. We follow the argument in [GG2]. Since $0<\sigma<1$ is the Hölder coefficient of the $A_{i j}^{\alpha \beta}$, then we have

$$
\omega(r) \leq C r^{\sigma / 2}
$$

Let $\Omega_{1} \subset \subset \Omega \backslash S$ and let $d=\frac{1}{2} d\left(\Omega_{1}, \partial(\Omega \backslash S)\right)$. Let $p_{0} \in \Omega_{1}$ and $R<$ $\min (1, d)$. Following the argument in Lemma 5.3, we split $u=v+w$ in $B\left(p_{0}, R\right)$. 
Using (3.23) we obtain for $0<r<R$, the estimate

$$
\begin{gathered}
\int_{B\left(p_{0}, r\right)}\left|X u-(X u)_{p_{0}, r}\right|^{2} d x \\
\leq C\left[\left(\frac{r}{R}\right)^{Q+2} \int_{B\left(p_{0}, R\right)}\left|X u-(X u)_{p_{0}, R}\right|^{2} d x+\int_{B\left(p_{0}, R\right)}|X w|^{2} d x\right] .
\end{gathered}
$$

From (5.13), (5.16) and as a consequence of (5.15) we obtain

$$
\int_{B\left(p_{0}, R\right)}|X w|^{2} d x \leq C R^{Q-2+2 \alpha+\alpha \sigma \frac{(q-2)}{q}},
$$

for every $0<\alpha<1$. Consequently (5.17) yields

$$
\begin{gathered}
\int_{B\left(p_{0}, r\right)}\left|X u-(X u)_{p_{0}, r}\right|^{2} d x \\
\leq C\left[\left(\frac{r}{R}\right)^{Q+2} \int_{B\left(p_{0}, R\right)}\left|X u-(X u)_{p_{0}, R}\right|^{2} d x+R^{Q-2+2 \alpha+\alpha \sigma \frac{(q-2)}{q}}\right] .
\end{gathered}
$$

If $\alpha$ is close enough to 1 , so that $2 \alpha+\alpha \sigma \frac{(q-2)}{q}>2$, then using a standard argument (see Lemma 2.1 in [Gi], Chap. 3) we can readily deduce that $X u$ is Hölder continuous in $\Omega_{1}$ with some exponent. In particular, $X u$ is locally bounded in the same set. Now we want to prove that $X u$ is locally Hölder continuous in $\Omega_{1}$ with exponent $\sigma$. Using the boundedness of $X u$ in (5.8) along with the Poincare inequality, we can prove

$$
\int_{B\left(p_{0}, R\right)}|X w|^{2} d x \leq C R^{Q+2 \sigma} .
$$

The proof is concluded once we introduce the latter inequality in (5.17), and use the iteration lemma 2.1 in [Gi], Chap. 3.

For the scalar (diagonal) case the reader should consult [XZ], [C1] and [C2].

\section{References}

[AK1] L. Ambrosio, B. Kirchheim: Rectifiable sets in metric and Banach spaces, Math. Ann. 318, 527-555 (2000)

[AK2] L. Ambrosio, B. Kirchheim: Currents in metric and Banach spaces, Acta Math. 185, 1-80 (2000)

[BR] A. Bellaiche, J.-J. Risler: Sub-Riemannian Geometry, Progress in Mathematics, vol. 114, Basel: Birkhäuser (1996)

[BI] B. Bojarski, T. Iwaniec: Analytical foundations of the theory of quasi-conformal maps, Ann. Acad. Scie. Fenn, Series A 8, 257-254 (1993)

[Br] R.W. Brockett: Control theory and singular Riemannian geometry, New directions in applied mathematics (Cleveland, Ohio, 1980), pp. 11-27, New York-Berlin: Springer (1982)

[C1] L. Capogna: Regularity for quasilinear equations in the Heisenberg group, Comm. in Pure Appl. Math. 50, 867-889 (1997) 
[C2] L. Capogna: Regularity for quasilinear equations and 1-quasiconformal maps in Carnot groups, Math. Ann. 313(2), 263-295 (1999)

[C3] L. Capogna: PhD dissertation, Purdue University (1996)

[CG] L. Capogna, N. Garofalo: Boundary behavior of positive, solutions to sub-elliptic equations in NTA domains for Carnot-Caratheodory metrics, J. Fourier Anal. Appl. 4, 403-432 (1999)

[Ca] C. Carathéodory: Untersuchungen über die Grundlangen der Thermodynamik, Math. Ann. 67, 355-386 (1909)

[Che] J. Cheeger: Differentiability of Lipschitz functions on metric measure spaces, Geom. Funct. Anal. 9(3), 428-517 (1999)

[CK] G.S. Chirikjian, A.B. Kyatkin: Engineering Applications of Noncommutative Harmonic Analysis, Boca Raton, London, New York, Washington, D.C.: CRC Press (2001)

[Cho] W.L. Chow: Über Systeme von linearen partiellen Differentialgleichungen erster Ordnung, Math. Ann. 117, 98-105 (1939)

[Ch] M. Christ: A $T(b)$ theorem with remarks on analytic capacity and the Cauchy integral, Coll. Math. LX-LXI, 601-628 (1990)

[CGGP] M. Christ, D. Geller, P. Glowacki, L. Polin: Pseudodifferential operators on groups with dilations, Duke Math. J. 68, 31-65 (1992)

[Chr] D. Christodoulou: On the geometry and dynamics of crystalline continua, Ann. Inst. H. Poincaré 13, 335-358 (1998)

[CW] R.R. Coifman, G. Weiss: Analyse Harmonique Non-Commutative sur Certaines Espaces Homogènes; Étude de Certains Intégrales Singulières, Lecture Notes in Mathematics, vol. 242, Berlin-New York: Springer (1971)

[CoG] L. Corwin, F.P. Greenleaf: Representations of nilpotent Lie groups and their applications, Part I: basic theory and examples, Cambridge Studies in Advanced Mathematics 18, Cambridge: Cambridge University Press (1990)

[Cy] J. Cygan: Subadditivity of homogeneous norms on certain nilpotent Lie groups, Proc. Amer. Math. Soc. 83(1), 69-70 (1981)

[DP] G. Da Prato: Spazi $\mathcal{L}^{(p, \theta)}(\Omega, \delta)$ e loro proprietá, Ann. Mat. Pura Appl. 69, 383-392 (1965)

[E] L.C. Evans: Quasiconvexity and partial regularity in the calculus of variations, Arch. Rat. Mech. Anal. 95(3), 227-252 (1986)

[F] G.B. Folland: Subelliptic estimates and function spaces on nilpotent Lie groups, Ark. Math. 13, 161-207 (1975)

[FS] G.B. Folland, E.M. Stein: Estimates for the $\bar{\partial}_{b}$ complex and analysis on the Heisenberg group, Comm. Pure Appl. Math. 27, 459-522 (1974)

[FSC] B. Franchi, F. Serra Cassano: Gehring's lemma for metrics and higher integrability of the gradient for minimizers of noncoercive variational functionals, Studia Math. 120, 1-22 (1996)

[Ga] N. Garofalo: Recent Developments in the Theory of Subelliptic Equations and Its Geometric Aspects, Birkhäuser, to appear

[GN] N. Garofalo, D.-M. Nhieu: Isoperimetric and Sobolev inequalities for CarnotCarathéodory spaces and the existence of minimal surfaces, Comm. in Pure and Appl. Math. 49, 1081-1144 (1996)

[Ge] F.W. Gehring: The $L^{p}$ integrability of the partial derivatives of a quasiconformal map, Acta Math. 130, 265-277 (1973)

[G] D. Geller: Analytic Pseudodifferential Operators for the Heisenberg Group and Local Solvability, Princeton, NJ: Princeton University Press (1990)

[Gi] M. Giaquinta: Multiple integrals in the calculus of variations and nonlinear elliptic systems, Princeton, NJ: Princeton University Press (1983)

[GG1] M. Giaquinta, E. Giusti: Partial regularity for the solutions of nonlinear parabolic systems, Ann. Mat. Pura Appl. 97, 253-266 (1973)

[GG2] M. Giaquinta, E. Giusti: Nonlinear elliptic systems with quadratic growth, Man. Math. 24, 323-349 (1978) 
[GG3] M. Giaquinta, E. Giusti: On the regularity of minima of variational integrals, Acta Math. 148, 31-46 (1982)

[GMo] M. Giaquinta, G. Modica: Regularity results for some classes of higher order nonlinear elliptic equations, J. für Reine und Angew. Math. 311/312, 145-169 (1979)

[Giu] E. Giusti: Precisazione delle funzioni in $H^{1, p}$ e singolaritá nelle soluzioni deboli di sistemi ellittici non lineari, Boll. UMI 1, 71-76 (1969)

[GM] E. Giusti, M. Miranda: Sulla regolaritá nelle soluzioni deboli di una classe di sistemi ellittici nonlineari, Arch. Rat. Mech. Anal. 31, 173-184 (1968)

[Gr] M. Gromov: Carnot-Carathéodory spaces seen from within, Sub-Riemannian Geometry, ed. by A. Bellaiche, J.-J. Risler, Progress in Mathematics, vol. 114, pp. 79-323, Basel: Birkhäuser (1996)

[HK] P. Hajlasz, P. Koskela: Sobolev met Poincare, Mem. Amer. Math. Soc. 145 (2000)

[HS] P. Hajlasz, P. Strzelecki: Subelliptic $p$-harmonic maps into spheres and the ghost of Hardy spaces, Math. Ann. 312(2), 341-362 (1998)

[H] L. Hörmander: Hypoelliptic second order differential equations, Acta Math. 119, 147-171 (1967)

[J] D.S. Jerison: The Poincare inequality for vector fields satisfying Hörmander's condition, Duke Math J. 53, 503-523 (1986)

[JX] J. Jost, C.-J. Xu: Subelliptic harmonic maps, Trans. Amer. Math. Soc. 350(11), 4633-4694 (1998)

[K] J.J. Kohn: Pseudo-differential operators and hypoellipticity, Proceedings of Symposia in Pure Math. XXIII, 61-69 (1973)

[Ko] A. Kolmogorov: Zufällige Bewegungen, Ann. Math. 35, 116-117 (1934)

[Lu] G. Lu: The sharp Poincaré inequality for free vector fields: An endpoint result, Rev. Mat. Iberoamericana 10, 453-466 (1994)

[Mo] C.B. Morrey Jr.: Second order elliptic equations in several variables and Hölder continuity, Math. Z. 72, 146-164 (1959)

[Pa] P. Pansu: Métrique de Carnot-Carathëodory et quasiisométries des espaces symétriques de rang un, Annals of Math. 129(1), 1-60 (1989)

[Pe] J. Peetre: A theory of interpolation of normed spaces, Notas de Matématica, vol. 39, Rio de Janeiro: Instituto de Matématica pura e aplicada (1968)

[S] E.M. Stein: Harmonic Analysis: Real Variable Methods, Orthogonality and Oscillatory Integrals, Princeton: Princeton Univ. Press (1993)

[Su] H.J. Sussmann: Optimal control theory and piecewise analyticity of the distance function for some real-analytic sub-Riemannian metrics, Optimization and nonlinear analysis (Haifa, 1990), Pitman Res. Notes Math. Ser., vol. 244, pp. 298-310, Harlow: Longman Sci. Tech. (1992)

[T] M. Taylor: Noncommutative microlocal analysis, part I, Mem. Amer. Math. Soc. 52 (1984)

[Tr] H. Triebel: Interpolation theory, functions spaces, differential operators, Amsterdam-New York-Oxford: North-Holland (1978)

[Va] N.T. Varopoulos: Fonctions harmoniques sur les groupes de Lie, C.R. Acad. Sc. Paris 304, 519-521 (1987)

[V] Varadarajan V. S.: Lie Groups, Lie Algebras, and Their Representation, Prentice Hall (1974)

[XZ] C.-J. Xu, C. Zuily: Higher regularity for quasilinear subelliptic systems, Calc. of Variations and PDE's 5(4), 323-344 (1997) 